\title{
SIMPLE GROUPS ADMIT BEAUVILLE STRUCTURES
}

\author{
ROBERT GURALNICK AND GUNTER MALLE
}

Dedicated to the memory of Fritz Grunewald

\begin{abstract}
We answer a conjecture of Bauer, Catanese and Grunewald showing that all finite simple groups other than the alternating group of degree 5 admit unmixed Beauville structures. We also consider an analog of the result for simple algebraic groups which depends on some upper bounds for character values of regular semisimple elements in finite groups of Lie type. Finally, we prove that any finite simple group contains two conjugacy classes $C, D$ such that any pair of elements in $C \times D$ generates the group.
\end{abstract}

\section{INTRODUCTION}

A Beauville surface is a compact complex surface $S$ which is rigid (i.e., it has no nontrivial deformations) and satisfies $S=(X \times Y) / G$ where $X$ and $Y$ are curves of genus at least 2 and $G$ is a finite group acting freely on $X \times Y$. See [3] for more background on the history and importance of Beauville surfaces.

A finite group $G$ is said to admit an unmixed Beauville structure if there exist two pairs of generators $\left(x_{i}, y_{i}\right), i=1,2$, for $G$ such that $\Sigma\left(x_{1}, y_{1}\right) \cap \Sigma\left(x_{2}, y_{2}\right)=\{1\}$, where for $x, y \in G$ we set

$$
\Sigma(x, y)=\bigcup_{i \geq 0, g \in G}\left\{g x^{i} g^{-1}, g y^{i} g^{-1}, g(x y)^{i} g^{-1}\right\} .
$$

In particular, if there are two generating pairs $\left(x_{i}, y_{i}\right)$ such that the orders of $x_{1}, y_{1}$ and $x_{1} y_{1}$ are relatively coprime to those of $x_{2}, y_{2}$ and $x_{2} y_{2}$, then $G$ admits an unmixed Beauville structure. By the Riemann existence theorem, each generating pair $\left(x_{i}, y_{i}\right)$ of $G$ gives rise to a Galois action of $G$ on a curve $X_{i}$ such that $X_{i} \rightarrow X_{i} / G \cong \mathbb{P}^{1}$ is branched at 3 points. The condition that $\Sigma\left(x_{1}, y_{1}\right) \cap \Sigma\left(x_{2}, y_{2}\right)=\{1\}$ is precisely the condition that the action of $G$ on $X_{1} \times X_{2}$ is free.

Our first main result completes a program announced and started in [22] and answers a conjecture of Bauer-Catanese-Grunewald [2, 3] regarding Beauville structures. See also 14] for some low rank cases.

Theorem 1.1. Let $G$ be a finite non-abelian simple group other than $\mathfrak{A}_{5}$. Then $G$ admits an unmixed Beauville structure.

The proof will be given in Sections 24 ,

See Garion-Larsen-Lubotzky [16] for a proof that this holds for all sufficiently large simple groups of Lie type, and Fuertes-González-Diez [13, Thm. 1] for the alternating groups. Our proof is independent of the result in [16]. The statement for alternating groups also follows easily by [4].

Date: September 20, 2018.

The first author was partially supported by NSF grants DMS 0653873 and 1001962 . 
In the proof, the well-known character formula for structure constants in finite groups will be essential: Let $G$ be a finite group, $C_{1}, C_{2}, C_{3}$ conjugacy classes of $G$. Then for fixed $x \in C_{1}$ the number of pairs

$$
n\left(C_{1}, C_{2}, C_{3}\right):=\left|\left\{(y, z) \in C_{2} \times C_{3} \mid x y z=1\right\}\right|
$$

in $G$ is given by the character formula

$$
n\left(C_{1}, C_{2}, C_{3}\right)=\frac{\left|C_{2}\right| \cdot\left|C_{3}\right|}{|G|} \sum_{\chi \in \operatorname{Irr}(G)} \frac{\chi\left(C_{1}\right) \chi\left(C_{2}\right) \chi\left(C_{3}\right)}{\chi(1)},
$$

where the sum ranges over the complex irreducible characters of $G$ and $\chi\left(C_{i}\right)$ denotes the value of $\chi$ on elements of $C_{i}$.

If $G$ is simple of Lie type, the first two conjugacy classes contain regular semisimple elements and the third class non-identity semisimple elements, then the structure constant is always non-zero by the nice result of Gow [19, Thm. 2].

We will also use several results about maximal subgroups containing special elements (mostly based on Guralnick-Penttila-Praeger-Saxl [23]). We also obtain some new results that may be of independent interest about maximal subgroups of the exceptional groups, see Theorem 2.1. In general, the idea of the proof is fairly simple. We find three conjugacy classes $C_{i}$ of our simple group such that there are no maximal subgroups intersecting all three classes (or at least very few).

These ideas allow us to prove some related results. The first is:

Theorem 1.2. If $G$ is a simply connected simple algebraic group of rank $r>1$ over an algebraically closed field, and $C_{i}, 1 \leq i \leq 3$, are conjugacy classes of regular semisimple elements of $G$, then the variety $\left\{\left(x_{1}, x_{2}, x_{3}\right) \mid x_{i} \in C_{i}, x_{1} x_{2} x_{3}=1\right\}$ is irreducible of dimension $2 \operatorname{dim} G-3 r$.

We prove the result over the algebraic closure of a finite field by using our results on the finite groups of Lie type. From this, the theorem follows by a simple argument (as pointed out to us by Michael Larsen). See Theorem 6.5 and Remark 6.14. For the exceptional groups, we prove the analogous result with $C_{3}$ an almost arbitrary class. See Theorem 6.16 for the precise statement.

The ideas used in the proof also allow us to show (see Section 7):

Theorem 1.3. Let $G$ be a finite simple group. There exist conjugacy classes $C$ and $D$ of $G$ such that $G=\langle c, d\rangle$ for any $c \in C$ and $d \in D$.

In [10, Thm. A], finite solvable groups were characterized by the property that for any pair of conjugacy classes $C, D$, there exist $(c, d) \in C \times D$ with $\langle c, d\rangle$ solvable. Using a variation of the reduction to almost simple groups in [10] and a slight generalization of the previous result characterizes any family of finite groups closed under subgroups, quotients and extensions in a similar fashion. More precisely, one has the following result (see also [11, Thm. C]):

Corollary 1.4. Let $X$ be a family of finite groups closed under subgroups, quotients and extensions. A finite group $G$ belongs to $X$ if and only if for every $x, y \in G,\left\langle x, y^{g}\right\rangle \in X$ for some $g \in G$. 
The paper is organized as follows. In the next three sections, we prove Theorem 1.1 for exceptional groups, classical groups and sporadic groups.

In Section 5, we prove Theorem 5.4 which gives an upper bound for the absolute value of character values on semisimple elements in groups of Lie type (including disconnected groups - see [16, Thm. 3] for a different proof with a larger bound). We then use this result to study the structure of the variety of triples of elements in three semisimple regular classes with product 1 .

In the final section, we prove a slightly more general version of Theorem 1.3 that allows one to obtain Corollary 1.4.

Acknowledgement. We wish to thank M. Larsen and T. Springer for various remarks about the results of this paper. In particular, their comments allowed us to extend the proof of Theorem 1.2 to all semisimple regular classes and to characteristic 0 and to prove the irreducibility of the variety.

Remark. After this paper was completed and posted, we were informed by Fairbairn, Magaard and Parker that they also proved Theorem 1.1] using similar methods (see [12]). Also, Kantor, Lubotzky and Shalev have just sent us a preprint [27] including a proof of Theorem 1.3 .

\section{EXCEPTIONAL GROUPS}

Here, we show Theorem 1.1 for the exceptional groups of Lie type; the Tits group ${ }^{2} F_{4}(2)^{\prime}$ will be considered in Proposition 4.1. We also exclude the solvable group ${ }^{2} B_{2}(2)$ and the non-simple groups with classical socle $G_{2}(2) \cong \mathrm{U}_{3}(3) .2$ and ${ }^{2} G_{2}(3) \cong \mathrm{L}_{2}(8) .3$ throughout this section.

First we prove a result on overgroups of certain maximal tori, which may be of independent interest. In the case of $E_{7}(q)$ this is due to Weigel [45, 4(i)].

Theorem 2.1. Let $G$ be a simple exceptional group of Lie type different from ${ }^{3} D_{4}(q)$. Then there exists a cyclic subgroup $T \leq G$ such that $|T|$ and the maximal overgroups $M \geq T$ in $G$ are as given in Table 1 .

In the table, $\Phi_{i}$ denotes the $i$ th cyclotomic polynomial evaluated at $q, \Phi_{8}^{\prime \prime}=q^{2}-\sqrt{2} q+1$, $\Phi_{12}^{\prime \prime}=q^{2}-\sqrt{3} q+1, \Phi_{24}^{\prime \prime}=q^{4}-\sqrt{2} q^{3}+q^{2}-\sqrt{2} q+1$. For $G_{2}(q), \epsilon \in\{ \pm 1\}$. In $E_{7}(q)$, $P, P^{\prime}$ denote two maximal $E_{6}$-parabolic subgroups, and $L$ their common Levi factor; $d=\operatorname{gcd}(3, q-1)$ for $E_{6}(q), d=\operatorname{gcd}(3, q+1)$ for ${ }^{2} E_{6}(q), d=\operatorname{gcd}(2, q-1)$ for $E_{7}(q)$.

Proof. The existence of maximal tori of the given orders follows from general theory, see [40, Prop. 25.3] for example. The maximal subgroups of the exceptional groups of Lie type of small rank are known explicitly, see [7, 30, 39]. From those lists, it is straightforward to check the first five lines of the table.

For $E_{7}(q)$ the claim is in [45, 4(i)]. For $F_{4}(2),{ }^{2} E_{6}(2)$ and $E_{6}(2)$ the maximal subgroups are listed in [6]. For the remaining exceptional groups of large rank, we use the results of Liebeck-Seitz [32]. Let $M$ be a maximal subgroup of $G$ containing $T$. By [32, Thm. 8], using Zsigmondy prime divisors of $|T|$, one finds that either $M$ is reductive of maximal rank as given in the table, or almost simple. In the latter case, by [32, Table 2] the socle $S$ of $M$ is of Lie type in the same characteristic as $G$. By [32, Thm. 8(VI)] the untwisted 
TABLE 1. Maximal overgroups of cyclic subgroups in exceptional groups

\begin{tabular}{|l||c|l|l|}
\hline$G$ & $|T|$ & $M \geq T$ & further maximal overgroups \\
\hline \hline${ }^{2} B_{2}\left(q^{2}\right), q^{2} \geq 8$ & $\Phi_{8}^{\prime \prime}$ & $T: 4$ & - \\
\hline${ }^{2} G_{2}\left(q^{2}\right), q^{2} \geq 27$ & $\Phi_{12}^{\prime \prime}$ & $T: 6$ & - \\
\hline$G_{2}(q), 3 \mid q-\epsilon$ & $q^{2}+\epsilon q+1$ & $\mathrm{SL}_{3}^{\epsilon}(q) .2$ & $q=4: J_{2}, G_{2}(2), \mathrm{L}_{2}(13)$ \\
\hline$G_{2}(q), 3 \mid q$ & $q^{2}-q+1$ & $\mathrm{SU}_{3}(q) .2(2 \times)$ & $q=3:{ }^{2} G_{2}(3), 2^{3} \cdot \mathrm{L}_{3}(2), \mathrm{L}_{2}(13)$ \\
\hline${ }^{2} F_{4}\left(q^{2}\right), q^{2} \geq 8$ & $\Phi_{24}^{\prime \prime}$ & $T: 12$ & - \\
\hline$F_{4}(q), 2 \nmid q$ & $\Phi_{8}$ & $\mathrm{Spin}_{9}(q)$ & - \\
\hline$F_{4}(q), 2 \mid q$ & $\Phi_{8}$ & $\mathrm{SO}_{9}(q)(2 \times)$ & - \\
\hline$E_{6}(q)$ & $\Phi_{3} \Phi_{12} / d$ & $\Phi_{3} \cdot{ }^{3} D_{4}(q) .3 / d$ & - \\
\hline${ }^{2} E_{6}(q)$ & $\Phi_{6} \Phi_{12} / d$ & $\Phi_{6} \cdot{ }^{3} D_{4}(q) .3 / d$ & $q=2: F_{4}(2), F i_{22}(3$ each $)$ \\
\hline$E_{7}(q)$ & $\Phi_{1} \Phi_{9} / d$ & $P, P^{\prime}, L \cdot 2$ & - \\
\hline$E_{8}(q)$ & $\Phi_{15}$ & $T: 30$ & - \\
\hline
\end{tabular}

rank of $S$ is at most half the rank of $G$, and either $S$ is defined over a field of order at most 9 , or it is ${ }^{\epsilon} \mathrm{L}_{3}(16)$ or of rank 1 . It ensues that the only possibilities are $S=\mathrm{S}_{4}(9)$ or $\mathrm{L}_{2}(81)$ inside $F_{4}(3)$.

So assume $G=F_{4}(3)$. The torus of order $q^{4}+1$ is contained in the subsystem subgroup $B_{4}(q)=\operatorname{Spin}_{9}(q)$, for which the 25-dimensional $G$-module $V$ has two composition factors: once the 16-dimensional spin-module and once the natural module for $\mathrm{SO}_{9}(q)$. Any regular element $x$ of order $q^{4}+1$ is conjugate to 8 of its powers, so it fixes a 1-dimensional subspace of $V$. First assume that $S=\mathrm{S}_{4}(9)$ embeds into $G$. By the theory of irreducible $\overline{\mathbb{F}}_{3} \mathrm{Sp}_{4^{-}}$ modules, the irreducible $\mathbb{F}_{3} S$-modules have dimension $1,10,16,20,25$ or bigger. Since $S$ can have at most one fixed point, this shows that $\left.V\right|_{S}$ is irreducible (in fact, absolutely irreducible). But by [33, Cor. 2 (Table 1.3)] there is no such irreducible embedding.

So now consider $S=\mathrm{L}_{2}(81)$. Again, it's easily seen that the irreducible $\mathbb{F}_{3} S$-modules have dimension 1,8,12,16 or bigger than 25 . Here, the 12-dimensional module cannot occur in the restriction $\left.V\right|_{S}$ since it is the sum of four Galois conjugates of the 3-dimensional orthogonal group, and thus the elements of order 41 have a 4-dimensional fixed space. Furthermore, by [1, Cor. 4.5] there are no non-trivial extensions between the trivial module and the tensor product of the natural $S$-module with its double Frobenius twist. Since $V$ is self-dual, this implies that $S$ has a 1-dimensional fixed space on $V$, so in fact $S$ is contained in the stabilizer of this one-space. But the stabilizer of the 1-space centralized by $x$ is a $\operatorname{Spin}_{8}(3)$, whence $S$ has to be contained in a subgroup $\operatorname{Spin}_{8}$ and cannot be maximal.

Theorem 2.2. Let $G$ be a simple exceptional group of Lie type different from ${ }^{3} D_{4}(q)$ and $C$ the conjugacy class of a generator of the cyclic subgroup given in Table 1 . Then there exist $x_{1}, x_{2}, x_{3} \in C$ with $G=\left\langle x_{1}, x_{2}\right\rangle$ and $x_{1} x_{2} x_{3}=1$.

Proof. The proof is very similar to that of our result in [22, Prop. 3.4 and 3.5]. We either compute the structure constant $n(C, C, C)$ in $G$ from the known generic character tables [17, or estimate it from below using [22, Prop. 3.3] to be at least $\frac{1}{2}|G| /|T|^{2}$. 
We illustrate this on one of the more difficult cases, viz. $E_{6}(q)$. All elements of $T$ whose order does not divide $\Phi_{3}$ are regular. The other non-central ones have centralizer $\Phi_{3} \cdot{ }^{3} D_{4}(q) / d$. Thus, by [22, Lemma 3.2] the irreducible characters of $H=E_{6}(q)_{\text {ad }}$ not vanishing on $C$ lie in Lusztig series $\mathcal{E}(H, s)$ where $s \in H^{*}=E_{6}(q)_{\text {sc }}$ either is regular of order dividing $\Phi_{3} \Phi_{12}$, or $s$ has centralizer $\Phi_{3} \cdot{ }^{3} D_{4}(q)$, or $s \in Z\left(H^{*}\right)$. The characters in the latter series are the extensions to $H$ of the unipotent characters of $G$. From the degree formulas in [5, 13.9] it follows that just 11 non-trivial unipotent characters do not vanish on $C$, and their values on $x \in C$ are \pm 1 . The characters corresponding to $s$ with centralizer $Z=\Phi_{3} \cdot{ }^{3} D_{4}(q)$ have degree at least $|H|_{p^{\prime}} /|Z|_{p^{\prime}} \geq q^{20}$ where $p$ is the defining prime of $G$. By [22, Prop. 3.3] values on $C$ are bounded above by 12 , and there are at most $q^{2}+q$ such characters. This gives an upper bound for their contribution to $n(C, C, C)$. Finally, the characters in $\mathcal{E}(H, s)$ with $s$ regular semisimple are irreducible Deligne-Lusztig characters, of degree $|H|_{p^{\prime}} /|T|_{p^{\prime}}$, which is roughly $q^{32}$, and there are less than $\Phi_{3} \Phi_{12} / 12$ of them. In conclusion, the contribution from the non-linear characters to the structure constant is (much) less than $1 / 2|G| /|T|^{2}$.

On the other hand, the maximal overgroups of $T$ are known by Theorem 2.1. Clearly, any subgroup $H$ contains at most $|H|$ triples from $C$ with fixed first component. So it suffices to check that the sum of orders of relevant maximal subgroups is less than $\frac{1}{2}|G| /|T|^{2}$. For example in $E_{7}(q)$, the $E_{6}$-parabolic subgroups have order roughly $q^{107}$, while $|G| /|T|^{2}$ is roughly $q^{119}$. In all other cases, the maximal subgroups are even smaller.

Proposition 2.3. The simple exceptional groups of Lie type admit an unmixed Beauville structure.

Proof. This is now immediate for all types but ${ }^{3} D_{4}(q)$. Indeed, for all exceptional simple groups $G$ we proved in [22, Thm. 1.1] the existence of a conjugacy class $C$ such that $G$ is generated by $x_{1}, y_{1} \in C$ with $\left(x_{1} y_{1}\right)^{-1} \in C$. Theorem 2.2 shows that there exists a second such class $C^{\prime}$, and it is immediate to verify that the elements in $C^{\prime}$ have order coprime to that of elements in $C$.

Thus, to prove the claim it will suffice to exhibit for $G={ }^{3} D_{4}(q)$ a second generating system $\left(x_{2}, y_{2}\right)$ such that the orders of $x_{2}, y_{2}, x_{2} y_{2}$ are prime to the common order $m(G)=$ $q^{4}-q^{2}+1$ of the elements in $C$.

For ${ }^{3} D_{4}(2)$ the $(7 d, 7 d, 9 a)$-structure constant is non-zero, and by [6] no maximal subgroup contains elements from both classes. So now assume $q>2$. We let $C_{1}$ be a conjugacy class of regular semisimple elements of order $\Phi_{3}$ inside a maximal torus of or$\operatorname{der} \Phi_{3}^{2}$ (such elements exist for all $q$, see [17]) and $C_{2}$ a class of regular semisimple elements of order $\Phi_{6} \Phi_{2} \Phi_{1}$ (such elements exist when $q \neq 2$ ).

By [29] the only maximal subgroups containing an element of order $\Phi_{3}$ with centralizer of order dividing $\Phi_{3}^{2}$ are $G_{2}(q), \mathrm{PGL}_{3}(q),\left(\Phi_{3} \circ \mathrm{SL}_{3}(q)\right) .2 d\left(\right.$ where $\left.d=\operatorname{gcd}\left(3, q^{2}+q+1\right)\right)$ and the torus normalizer $\Phi_{3}^{2} \cdot \mathrm{SL}_{2}(3)$. Now note that since $q>2$ none of these contains elements of order $\Phi_{6} \Phi_{2} \Phi_{1}$ (by Zsigmondy's theorem for the latter three groups, and since elements of order $\Phi_{6}$ in the first subgroup are self-centralizing). Thus any pair of elements $\left(x_{1}, x_{2}\right) \in C_{1} \times C_{2}$ generates. Using the result of Gow [19, Thm. 2] or the generic character table one sees that there exist such pairs with product in any non-trivial semisimple conjugacy class. 
Alternatively, it would have been possible to choose two classes of regular semisimple elements whose order is divisible by large Zsigmondy primes, and a further class containing the product of a long root element with a semisimple element of order divisible by a third Zsigmondy prime, in such a way that only the trivial character has non-zero value on all three classes. Then the character formula shows that the structure constant $n\left(C_{1}, C_{2}, C_{3}\right)$ equals $\left|C_{2}\right|\left|C_{3}\right| /|G|$ and in particular does not vanish.

From the enumeration of subgroups containing long root elements by Cooperstein it would then be easy to see that no triple in $C_{1} \times C_{2} \times C_{3}$ lies in a proper subgroup of $G$. This approach has been used in [22, Thm. 8.6] to show that $E_{8}(q)$ satisfies Theorem 1.1.

\section{Classical groups}

Here we prove Theorem 1.1 for the simple classical groups of Lie type.

The groups $\mathrm{L}_{2}(q), q \geq 7$ were shown to admit a Beauville structure in [2]. Before treating the generic case it will be convenient to consider some linear, unitary and symplectic groups of small rank.

Proposition 3.1. Theorem 1.1 holds for the simple groups $\mathrm{L}_{3}(q), \mathrm{U}_{3}(q)$ and $\mathrm{S}_{4}(q)$, where $q \geq 3$.

Proof. Let first $G=\mathrm{L}_{3}(q)$. Since $\mathrm{L}_{3}(2) \cong \mathrm{L}_{2}(7)$ we may assume that $q \geq 3$. In $[22$, Prop. 3.13] we showed that $G$ can be generated by a pair of elements of order $\Phi_{3}^{*}(q)$, with product of the same order. Let $C_{1}$ be the conjugacy class of a regular element $x$ of order $\left(q^{2}-1\right) / d$, with $d=\operatorname{gcd}(3, q-1)$. Then the only maximal subgroups containing $x$ are maximal parabolic, and three subgroups $\mathfrak{A}_{6}$ when $q=4$. Let $C_{2}$ consist of regular unipotent elements. Then the $\left(C_{1}, C_{2}, C_{2}\right)$-structure constant is non-zero in $G$ by [17]. Let $P$ be a maximal parabolic subgroup. Then its derived subgroup contains all unipotent elements, but no element of order $\left(q^{2}-1\right) / d$. Thus, $P$ cannot contain triples from $C_{1} \times$ $C_{2} \times C_{2}$ with product 1 . For $\mathrm{L}_{3}(4)$ it is easy to check that the structure constant in $G$ is larger than those in the $\mathfrak{A}_{6}$-subgroups.

Similarly, we showed in [22, Prop. 3.11] that $G=\mathrm{U}_{3}(q)$ with $q \geq 3$ can be generated by elements of order $\Phi_{6}^{*}(q)$. Again, let $C_{1}$ be a class of elements of order $\left(q^{2}-1\right) / d$, where $d=\operatorname{gcd}(3, q+1)$. The only maximal subgroups containing such elements are the Borel subgroups and the image in $G$ of a subgroup $\mathrm{GU}_{2}(q)$ of $\mathrm{SU}_{3}(q)$. Moreover, for $q=3,4,5$ there is an additional class of subgroups $4^{2} \cdot \mathfrak{S}_{3}, 5^{2} \cdot \mathfrak{S}_{3}$ respectively three classes of $M_{10}$.

Choosing $C_{2}$ to consist of regular unipotent elements, one checks that the $\left(C_{1}, C_{2}, C_{2}\right)$ structure constant is non-zero [17]. By quotienting out the normal closure of a Sylow $p$-subgroup we see that such triples cannot lie in either of the two generic maximal subgroups. For $q=3,4,5$ direct computation in $G$ shows that there exist generating triples.

For $G=\mathrm{S}_{4}(q), q \geq 3$ we produced in [22, Prop. 3.8] a generating triple with elements of order $\Phi_{4}^{*}(q)$. A direct computation shows that $\mathrm{S}_{4}(3)$ contains generating triples with orders $(9,9,8)$, and these are prime to $\left(3^{2}+1\right) / 2=5$. For $q \geq 4$ let $C_{1}$ denote a class of regular elements of order $(q+1) / d$ inside a maximal torus of order $(q+1)^{2} / d$, where $d=\operatorname{gcd}(2, q-1)$, and $C_{2}$ a class of regular unipotent elements. It follows from the known character table [43] that $n\left(C_{1}, C_{2}, C_{2}\right)>\frac{1}{4} q^{6}$. On the other hand, by [26, Thm. 5.6] for example, the only maximal subgroups of $G$ containing elements from class $C_{1}$ are the normalizers of subgroups $\mathrm{SO}_{4}^{+}(q)$ and $\mathrm{O}_{3}(q) \times \mathrm{O}_{2}^{-}(q)$. In the latter, any unipotent element 
is centralized by a 1-dimensional torus, so it does not contain regular unipotent elements. The structure constant in $H=\mathrm{SO}_{4}^{+}(q)$ can be estimated from above by the number of regular unipotent elements of $G$ contained in $H$, which is less than $2 q^{4}$. So there exist generating triples.

TABle 2. Maximal tori in some linear and unitary groups

\begin{tabular}{|ll||lll|}
\hline$G$ & {$[22]$} & $T_{1}$ & $T_{2}$ & $T_{3}$ \\
\hline \hline $\mathrm{SL}_{4}(q)$ & $\Phi_{3}^{*}$ & $\left(q^{2}+1\right)(q+1)$ & $\left(q^{2}-1\right)(q+1)$ & $\left(q^{2}-1\right)(q-1)$ \\
$\mathrm{SU}_{4}(q)$ & $\Phi_{6}^{*}$ & $\left(q^{2}+1\right)(q-1)$ & $\left(q^{2}-1\right)(q-1)$ & $\left(q^{2}-1\right)(q+1)$ \\
$\mathrm{SU}_{5}(q)$ & $\Phi_{10}^{*}$ & $q^{4}-1$ & $\left(q^{3}+1\right)(q-1)$ & $\left(q^{3}+1\right)(q+1)$ \\
$\mathrm{SU}_{6}(q)$ & $\Phi_{10}^{*}$ & $\Phi_{1} \Phi_{3} \Phi_{6}$ & $\Phi_{1}^{2} \Phi_{2} \Phi_{4}$ & $\Phi_{2} \Phi_{6}^{2}$ \\
\hline
\end{tabular}

Proposition 3.2. Theorem 1.1 holds for the simple groups $\mathrm{L}_{4}(q), \mathrm{U}_{4}(q), \mathrm{U}_{5}(q)$ and $\mathrm{U}_{6}(q)$.

Proof. For $G=\mathrm{SL}_{4}(q)$ we may assume that $q>3$ since $\mathrm{SL}_{4}(2) \cong \mathfrak{A}_{8}$ is an alternating group and, by explicit computation, $\mathrm{L}_{4}(3)$ has generating triples of elements of order 5 . Let $C_{i}$ be the class of a regular semisimple element $x_{i}$ in a maximal torus $T_{i}$ as in Table 2, $i=1,2,3$. Such classes exist whenever $q \geq 4$. Then $x_{1}$ acts irreducibly, and there exists a Zsigmondy prime for $o\left(x_{1}\right)$, so the maximal subgroups containing a triple $\left(x_{1}, x_{2}, x_{3}\right) \in$ $C_{1} \times C_{2} \times C_{3}$ are described in [22, Thm. 2.2]. The only ones containing Singer cycles are the normalizers of $\mathrm{GL}_{2}\left(q^{2}\right) \cap \mathrm{SL}_{4}(q)$ and $\mathrm{GU}_{2}\left(q^{2}\right) \cap \mathrm{SL}_{4}(q)$. But in the first group, any semisimple element has centralizer order divisible by either $\left(q^{4}-1\right) /(q-1)$ or by $(q+1)\left(q^{2}-1\right)$, while $x_{3}$ has only centralizer order $(q-1)\left(q^{2}-1\right)$. Similarly we can exclude the second case using the order of $x_{2}$. Thus any triple $\left(x_{1}, x_{2}, x_{3}\right)$ generates $G$. By [19, Thm. 2] there exist such triples with product 1.

The element orders are coprime to those of the element order chosen in [22, Prop. 3.13], so the Beauville property is satisfied, and we conclude by passing to the central quotient $G / Z(G)=\mathrm{L}_{4}(q)$.

For $G=\mathrm{SU}_{4}(q)$ regular elements as indicated in Table 2 exist whenever $q \geq 4$. The argument is then completely analogous; again the only subgroups possibly containing elements from $C_{1}$ is the normalizer of $\mathrm{GL}_{2}\left(q^{2}\right) \cap \mathrm{SU}_{4}(q)$ and the stabilizer of an isotropic point (a maximal parabolic subgroup with Levi factor $\mathrm{GL}_{2}\left(q^{2}\right) \cap \mathrm{SU}_{4}(q)$ ), which can be excluded as before. The group $\mathrm{SU}_{4}(2) \cong \mathrm{S}_{4}(3)$ was treated in Proposition 3.1, the group $\mathrm{SU}_{4}(3)$ has generating triples of elements of order 20.

For $G=\mathrm{SU}_{5}(q)$ choose $C_{i}$ to contain regular elements from the tori $T_{i}$ in Table 2 , which exist whenever $q \geq 3$. Here the only maximal subgroups of order divisible by $o\left(x_{i}\right)$, $i=1,2,3$, are $\mathrm{GU}_{4}(q)$. But there the centralizer order of a semisimple element of order $q^{3}+1$ is divisible by $\left(q^{3}+1\right)(q+1)$, while the element $x_{3}$ has smaller centralizer order $\left(q^{3}+1\right)(q-1)$. We now conclude as before. The group $\mathrm{SU}_{5}(2)$ has generating triples of order 15 .

For $G=\mathrm{SU}_{6}(q)$ we again choose $C_{i}$ to contain regular elements from the tori $T_{i}$ in Table 2, which exist whenever $q \geq 3$. Here the only maximal subgroups of order divisible by $o\left(x_{i}\right), i=1,2,3$, are the normalizers of $\mathrm{GL}_{3}\left(q^{2}\right) \cap \mathrm{SU}_{6}(q)$. But there the centralizer 
order of a semisimple element of order $q^{3}+1$ is divisible by $\left(q^{6}-1\right) /(q+1)$, while the element $x_{3}$ has centralizer order $\left(q^{3}+1\right)^{2} /(q+1)$. We now conclude as before. The group $\mathrm{SU}_{6}(2)$ has generating triples of order 10. Again, the element orders are coprime to those in [22], so the Beauville property is satisfied.

For the remaining classical groups, we choose conjugacy classes $C_{1}, C_{2}$ of regular semisimple elements of orders as given in Table [3. Here $k^{\epsilon}$ is shorthand for $q^{k}-\epsilon 1$, and $k^{\epsilon} \oplus(n-k)^{\delta}$ denotes an element which acts as $k^{\epsilon}$ on a subspace of dimension $k$ and as $(n-k)^{\delta}$ on a complementary subspace of dimension $n-k$ in the linear and unitary case, respectively twice the dimensions in the symplectic and orthogonal cases. We have also indicated the orders of elements in the generating triple constructed in [22]. It is straightforward to check that the cyclic subgroups chosen here intersect those from [22] trivially.

TABLE 3. Elements in classical groups

\begin{tabular}{|ll||l|ll|}
\hline$G$ & & {$[22]$} & $x_{1}$ & $x_{2}$ \\
\hline \hline $\operatorname{SL}_{n}(q)$ & $n \geq 5$ odd & $\Phi_{n}^{*}$ & $(n-1)^{+}$ & $(n-2)^{+} \oplus 2^{+}$ \\
& $n \geq 6$ even & $\Phi_{n-1}^{*}$ & $(n-2)^{+}$ & $(n-3)^{+} \oplus 3^{+}$ \\
\hline $\operatorname{SU}_{n}(q)$ & $n \geq 7$ odd & $\Phi_{2 n}^{*}$ & $(n-1)^{+}$ & $(n-4)^{-} \oplus 4^{+}$ \\
& $n \geq 8$ even & $\Phi_{2 n-2}^{*}$ & $(n-3)^{-} \oplus 3^{-}$ & $(n-5)^{-} \oplus 5^{-}$ \\
\hline $\operatorname{Spin}_{7}(q)$ & & $\Phi_{6}^{*}$ & $3^{+}$ & $2^{-} \oplus 1^{+}$ \\
$\operatorname{Spin}_{2 n+1}(q)$ & $n \geq 4$ & $\Phi_{2 n}^{*}$ & $(n-2)^{-} \oplus 2^{-}$ & $(n-3)^{-} \oplus 3^{+}$ \\
\hline $\operatorname{Sp}_{2 n}(q)$ & $n \geq 3$ & $\Phi_{2 n}^{*}$ & $n^{+}$ & $(n-1)^{-} \oplus 1^{-}$ \\
\hline $\operatorname{Spin}_{8}^{+}(q)$ & & $3^{-}$ & $4^{+}$ & $4^{+}$ \\
$\operatorname{Spin}_{10}^{+}(q)$ & & $4^{-}$ & $3^{-} \oplus 2^{-}$ & $5^{+}$ \\
$\operatorname{Spin}_{2 n}^{+}(q)$ & $n \geq 6$ & $(n-1)^{-}$ & $(n-2)^{-} \oplus 2^{-}$ & $(n-3)^{-} \oplus 3^{-}$ \\
\hline $\operatorname{Spin}_{2 n}^{-}(q)$, & $n \geq 4$ & $\Phi_{2 n}^{*}$ & $(n-1)^{-}$ & $(n-3)^{-} \oplus 3^{+}$ \\
\hline
\end{tabular}

In order to verify generation in classical groups, the following result will be useful and may be of independent interest. It follows by the main result of [23] and inspection of the tables in that paper.

Theorem 3.3. Let $G=\mathrm{GL}(V)=\mathrm{GL}_{n}(q)$ where $q=p^{a}$ with $p$ prime. Assume that $n>4$. Suppose that $H$ is an irreducible subgroup of $G$ containing elements of orders $r_{i}$, $i=1,2$ where $r_{i}$ is a Zsigmondy prime divisor of $q^{e_{i}}-1$ where $e_{1}>e_{2}>n / 2$. Then one of the following holds:

(1) $H$ contains $\mathrm{SL}(V)$, $\mathrm{SU}(V), \Omega^{( \pm)}(V)$ or $\mathrm{Sp}(V)$;

(2) $H$ preserves an extension field structure on $V$ (of degree $f$ dividing $\operatorname{gcd}\left(n, e_{1}, e_{2}\right)$ );

(3) $H$ normalizes $\mathrm{GL}_{n}\left(p^{b}\right)$ for some $b$ properly dividing a;

(4) $H \leq \mathrm{GL}_{1}(q)>\mathfrak{S}_{n}$ is imprimitive;

(5) H normalizes $\mathfrak{A}_{n+1+\delta}$ where $\delta=1$ if $\operatorname{gcd}(p, n) \neq 1$ and 0 otherwise;

(6) $n=5, H=M_{11},\left(e_{1}, e_{2}\right)=(5,4)$ and $q=3$;

(7) $n=6, H=2 . M_{12},\left(e_{1}, e_{2}\right)=(5,4)$ and $q=3$;

(8) $n=6$ or $7,\left(e_{1}, e_{2}\right)=(6,4)$ and $q$ is prime; or 
(9) $n=11, H=M_{23}$ or $M_{24},\left(e_{1}, e_{2}\right)=(11,10)$ and $q=2$;

We can get rid of some of these examples with a stronger hypothesis. This follows by the previous result and the computation of $\Phi_{e_{i}}^{*}(q)$, using [22, Lemma 2.1], for example:

Corollary 3.4. Let $G=\mathrm{GL}(V)=\mathrm{GL}_{n}(q)$ where $q=p^{a}$ with $p$ prime. Assume that $n>4$. Suppose that $H$ is an irreducible subgroup of $G$ containing elements of orders $\Phi_{e_{i}}^{*}(q)>1, i=1,2$ where $e_{1}>e_{2}>n / 2$. Then one of the following holds:

(1) $H$ contains $\mathrm{SL}(V), \mathrm{SU}(V), \Omega^{( \pm)}(V)$ or $\mathrm{Sp}(V)$;

(2) $H$ preserves an extension field structure on $V$ (of degree $f$ dividing $\operatorname{gcd}\left(n, e_{1}, e_{2}\right)$ );

(3) $H$ normalizes $\mathrm{GL}_{n}\left(p^{b}\right)$ for some $b$ properly dividing $a$;

(4) $n=6, H=2 . \mathrm{L}_{3}(4),\left(e_{1}, e_{2}\right)=(6,4)$ and $q=3$;

(5) $n=7, H=\operatorname{Sp}_{6}(2),\left(e_{1}, e_{2}\right)=(6,4)$ and $q=3$; or

(6) $H$ normalizes $\mathfrak{A}_{n+1+\delta}$ where $\delta=1$ if $\operatorname{gcd}(p, n) \neq 1$ and 0 otherwise, or $H \leq$ $\mathrm{GL}_{1}(q)>\mathfrak{S}_{n}$ is imprimitive, and either

(a) $q=2,\left(e_{1}, e_{2}\right) \in\{(12,10),(18,10),(18,12)\}$ and $e_{1} \leq n<2 e_{2}$; or

(b) $q=3,\left(e_{1}, e_{2}\right)=(6,4)$ and $n=6,7$.

Proposition 3.5. Theorem 1.1 holds for the linear groups $\mathrm{L}_{n}(q), n \geq 5$.

Proof. We argue in $G=\mathrm{SL}_{n}(q)$. We choose conjugacy classes $C_{1}, C_{2}$ in $G$ of regular semisimple elements $x_{i}$ of orders as given in Table 3, and we let $C_{3}$ be any class of semisimple elements of order prime to $\Phi_{n}^{*}(q)$ when $n$ is odd, respectively to $\Phi_{n-1}^{*}(q)$ when $n$ is even. First assume that Zsigmondy primes $r_{i}$ exist for the factors $\Phi_{e_{i}}(q)$ of $o\left(x_{i}\right)$ with $e_{i}>n / 2, i=1,2$. Then under each $x_{i} \in C_{i}, i=1,2$, the natural module of $G$ splits into two irreducible submodules of incompatible dimensions, so the subgroup $H:=\left\langle x_{1}, x_{2}\right\rangle$ generated by any pair of elements $x_{i} \in C_{i}$ is irreducible. We claim that $H=G$. Otherwise, when $n \neq 6$ we are in one of the cases of Corollary 3.4. Now note that $H$ cannot be an extension field subgroup since $\operatorname{gcd}\left(e_{1}, e_{2}\right)=1$, and it can't be a subfield subgroup by looking at a suitable Zsigmondy prime divisor of $o\left(x_{i}\right)$. Also, no proper classical subgroup has order divisible by both $o\left(x_{1}\right)$ and $o\left(x_{2}\right)$. Since $e_{2}=e_{1}-1$, we're not in cases $(4)-(6)$.

When $n=6$ then $e_{2}=n / 2$, so Corollary 3.4 is not applicable. Still, by [22, Lemma 2.1 and Thm. 2.2] we get the same conclusion as before unless $(n, q)=(6,2)$. In the latter case replace the second class by an irreducible element of order 63 and let $C_{3}$ consist of elements of order 7 with a 3-dimensional fixed space. By inspection of the possible overgroups in [23] the $x_{i}$ generate. In the Zsigmondy exception $(n, q)=(7,2)$, replace $C_{1}$ by the class of a regular element of type $4^{+} \oplus 3^{+}$; when $(n, q)=(8,2)$, replace it by the class of a Singer cycle, of order $\left(q^{8}-1\right) /(q-1)$. Then the previous arguments apply.

By [19, Thm. 2] there exists $x_{3} \in C_{3}$ with $x_{1} x_{2} x_{3}=1$. Now consider the image of this triple in the simple group $\mathrm{L}_{n}(q)$. The element orders are coprime to that for the generating triple exhibited in [22, Prop. 3.13] which proves the existence of a Beauville structure.

Proposition 3.6. Theorem 1.1 holds for the unitary groups $\mathrm{U}_{n}(q), n \geq 7$.

Proof. As before, we work in $G=\mathrm{SU}_{n}(q)$ and let $C_{1}, C_{2}$ contain regular semisimple elements $x_{1}, x_{2}$ of the orders indicated in Table 3 . For $n=8$ replace $C_{2}$ by a class 
of regular semisimple elements of order $\left(q^{8}-1\right) /(q+1)$. For $n=7$ let $C_{3}$ be a class of regular semisimple elements of type $5^{-} \oplus 2^{+}$, and otherwise let it be any class of semisimple elements of order prime to $\Phi_{2 n}^{*}(q)$ when $n$ is odd, respectively to $\Phi_{2 n-2}^{*}(q)$ when $n$ is even. Then $H=\left\langle x_{1}, x_{2}, x_{3}\right\rangle$, with $x_{i} \in C_{i}$, acts irreducibly on the natural module for $G$. For each $i$ let $e_{i}$ be maximal with the property that $o\left(x_{i}\right)$ has a corresponding Zsigmondy prime. Then $e_{i}>n$ for at least one $i$. Thus, if $H$ is proper then by [22, Thm. 2.2] either the Zsigmondy primes for both factors are small or the possible overgroups are classical, extension or subfield groups. The latter three classes can be excluded by using the fact that we have two distinct Zsigmondy primes. The first situation only arises when $(n, q) \in\{(7,2),(8,2)\}$ by [22, Lemma 2.1], but the conclusion still holds by [23].

So $H=G$ in all cases. Now the existence of triples $\left(x_{1}, x_{2}, x_{3}\right) \in C_{1} \times C_{2} \times C_{3}$ with product 1 follows from [19]. Passing to the quotient $\mathrm{U}_{n}(q)$ we obtain the desired result, noting again that the element orders are coprime to that in [22, Prop. 3.12].

Proposition 3.7. Theorem 1.1 holds for the symplectic groups $\mathrm{S}_{2 n}(q), n \geq 3$, q odd.

Proof. Let $G=\operatorname{Sp}_{2 n}(q), C_{1}$ and $C_{2}$ conjugacy classes of regular semisimple elements as indicated in Table 3 and $C_{3}$ any class of semisimple elements of order prime to $\Phi_{2 n}^{*}(q)$. Then for any pair $\left(x_{1}, x_{2}\right) \in C_{1} \times C_{2}$ of elements, the subgroup $H:=\left\langle x_{1}, x_{2}\right\rangle$ acts irreducibly on the natural module. If $H$ is proper, then by [22, Lemma 2.1 and Thm. 2.2], either $(n, q)=(4,3)$ or $H$ is contained in an extension or subfield subgroup. The latter cases do not occur by consideration of suitable Zsigmondy prime divisors. When $(n, q)=$ $(4,3)$, we choose $C_{3}$ to be a class of elements of order divisible by $q^{3}-1$; its Zsigmondy prime 13 gives no exception to [22, Thm. 2.2]. So we have $H=G$ in all cases. By [19] there exist triples from the chosen conjugacy classes with product 1.

In [22, Prop. 3.8] we produced a generating triple for $\mathrm{S}_{2 n}(q)$ consisting of elements of order $\Phi_{2 n}^{*}(q)$. This is coprime to the orders of $x_{1}, x_{2}$, so the proof is complete.

Proposition 3.8. Theorem 1.1 holds for the orthogonal groups $\mathrm{O}_{2 n+1}(q), n \geq 3$.

Proof. Let $G=\operatorname{Spin}_{2 n+1}(q), C_{1}, C_{2}$ conjugacy classes in $G$ of regular semisimple elements of orders as given in Table 3 and $C_{3}$ any class of semisimple elements of order prime to $\Phi_{2 n}^{*}(q)$, respectively a class of elements of order $q^{n-1}+1$ when $4 \leq n \leq 6$. For any pair $\left(x_{1}, x_{2}\right) \in C_{1} \times C_{2}$ of elements, the subgroup $H:=\left\langle x_{1}, x_{2}\right\rangle$ either acts irreducibly on the natural module, or it has a composition factor of dimension $2 n$. In the latter case, $H$ is contained in the stabilizer of an anisotropic line, so in a $2 n$-dimensional orthogonal group. But the first element does not lie in an orthogonal group of minus type, the second not in one of plus type, unless $n=3$ and $q \leq 4$. We return to these cases later. So otherwise $H$ is irreducible. Now note that for $(n, q) \neq(4,2)$, one of the two (respectively three when $n=4,5,6)$ element orders is divisible by a Zsigmondy prime divisor of $q^{e}-1$ with $2 e>2 n+1$. Moreover, for $n \geq 5$ there are even two different such $e$. If $H$ is proper, then by [22, Lemma 2.1 and Thm. 2.2] respectively Corollary 3.4 either $H$ is contained in an extension or subfield subgroup or we have $(n, q) \in\{(3,2),(4,2),(4,3),(8,2)\}$. Since none of the groups in Corollary 3.4(6) (a) contains elements of order $2^{12}-1,(n, q)=(8,2)$ is no exception. The other three groups will be considered later. The extension and subfield subgroups can be excluded by using suitable Zsigmondy primes.

In [22, Prop. 3.8] we produced a generating triple for $G=\mathrm{O}_{2 n+1}(q)$ consisting of elements of order $\Phi_{2 n}^{*}(q)$, which is prime to the orders chosen here. 
Finally, consider $\mathrm{O}_{7}(q)$ with $q \leq 4$ and $\mathrm{O}_{9}(q)$ with $q \leq 3$. Explicit computation shows that $\mathrm{O}_{7}(2)=\mathrm{S}_{6}(2)$ contains generating triples of order $7, \mathrm{O}_{7}(3)$ contains generating triples of order 13 , and $\mathrm{O}_{7}(4)=\mathrm{S}_{6}(4)$ contains generating triples of order 17 . The group $\mathrm{O}_{9}(2)=\mathrm{S}_{8}(2)$ contains generating triples of order $7, \mathrm{O}_{9}(3)$ contains generating triples of order 13 .

Proposition 3.9. Theorems 1.1 holds for the orthogonal groups $\mathrm{O}_{2 n}^{-}(q), n \geq 4$.

Proof. Let $G=\Omega_{2 n}^{-}(q)$. Let $C_{1}, C_{2}$ consist of regular semisimple elements of types as in Table 3 and $C_{3}$ any class of semisimple elements of order prime to $\Phi_{2 n}^{*}(q)$. Then any pair of elements $x_{i} \in C_{i}, i=1,2$, necessarily generates an irreducible subgroup $H:=\left\langle x_{1}, x_{2}\right\rangle$ of $G$, unless possibly when $n=4$. But in the latter case it is easy to see that no reducible subgroup has order divisible by Zsigmondy primes for $q^{3}+1$ and for $q^{3}-1$, which both exist when $q \neq 2$. We exclude $(n, q)=(4,2)$ for the moment. Otherwise, at least one of the two element orders $o\left(x_{i}\right)$ is divisible by a Zsigmondy prime divisor of $q^{e}-1$ with $e>n$. Moreover, for $n \geq 7$ there are even two different such $e$. Thus by [22, Lemma 2.1 and Thm. 2.2] and Corollary 3.4 we have $H=G$ unless $(n, q) \in\{(5,2),(6,2),(4,4)\}$. In the latter cases, let $C_{3}$ contain elements of order divisible by $2^{5}-1$ when $n=5,6$, by $4^{2}+1$ when $(n, q)=(4,4)$, then we still have generation for any triple $\left(x_{1}, x_{2}, x_{3}\right) \in C_{1} \times C_{2} \times C_{3}$. By [19], we can find $x_{i} \in C_{i}$ with product 1 .

The previously excluded group $\mathrm{O}_{8}^{-}(2)$ is generated by a triple from $(21 a, 21 a, 30 a)$. Combining this with [22, Prop. 3.6] the claim follows as in the previous cases.

Proposition 3.10. Theorem 1.1 holds for the orthogonal groups $\mathrm{O}_{2 n}^{+}(q), n \geq 4$.

Proof. We argue in $G=\Omega_{2 n}^{+}(q)$. First assume that $n>6$. Let $C_{1}$ consist of elements with precisely two invariant subspaces of dimensions 4 and $2 n-4$ and $C_{2}$ consist of elements with precisely two invariant subspaces of dimensions 6 and $2 n-6$. Moreover, assume that the orders of the elements in $C_{i}$ are divisible by all Zsigmondy prime divisors of $q^{2 n-4}-1$ and $q^{2 n-6}-1$, respectively. Let $C_{3}$ be any class of semisimple elements of order prime to $q^{n-1}+1$. By Corollary 3.4, there are no maximal subgroups containing elements from both $C_{1}$ and $C_{2}$. By [19], we can choose $x_{i} \in C_{i}$ with product 1 . Argue as usual to complete the proof.

If $n=6$, let $C_{1}$ and $C_{2}$ be as above. Now we apply [22, Thm. 2.2] instead and argue the same way as long as there is a Zsigmondy prime divisor $r$ of $q^{8}-1$ with $r>17$. This only fails for $q=2$. It can be checked with GAP that $\mathrm{O}_{12}^{+}(2)$ has a generating triple consisting of elements of order 17. Argue as above to complete the proof.

If $n=5$, let $C_{1}$ be as above. Let $C_{2}$ consist of elements of order $\left(q^{5}-1\right) /(2, q-1)$ and $C_{3}$ any class of semisimple elements of order prime to $q^{4}+1$. Apply [22, Thm. 2.2] to conclude that there are no maximal subgroups intersecting both $C_{1}$ and $C_{2}$ unless possibly $q \leq 5$. If $q=5$, inspection of the maximal subgroups shows the result is still true. By explicit computation the group $\mathrm{O}_{10}^{+}(2)$ contains generating triples of elements of order 31 , the group $\mathrm{O}_{10}^{+}(3)$ has generating triples of order 121, the group $\mathrm{O}_{10}^{+}(4)$ has generating triples of order 341. Argue as above to complete the proof.

If $n=4$ and $q>2$, let $C_{1}$ be a conjugacy class of regular semisimple elements of order $\left(q^{4}-1\right) /(2, q-1)$. Let $C_{2}$ and $C_{3}$ be the twists of $C_{1}$ by the triality automorphism and its square. By Kleidman [28], one sees that no maximal subgroup of $\mathrm{O}_{8}^{+}(q)$ intersects each 
of the $C_{i}$. By [19], there exist $x_{i} \in C_{i}$ with product 1 and they generate by the previous remarks.

By explicit computation the group $\mathrm{O}_{8}^{+}(2)$ contains generating triples of elements of order 7 .

In [22], we showed that there are generating triples of elements of $\mathrm{O}_{2 n}^{+}(q)$ in a class $C$ of regular semisimple elements of order dividing $\Phi_{2 n-2}^{*}(q)(q+1)$ lying in a maximal torus of order $\left(q^{n-1}+1\right)(q+1) / \operatorname{gcd}\left(4, q^{n-1}+1\right)$. Comparing the fixed spaces of elements in that torus with those in classes $C_{1}, C_{2}$ we conclude that the required intersection property holds.

\section{Alternating And Sporadic Groups}

The existence of unmixed Beauville structures for all alternating groups $\mathfrak{A}_{n}$ with $n \geq 6$ was proved by Fuertes-González-Diez [13, Thm. 1] after asymptotic results had been obtained by Bauer-Catanese-Grunewald 2]. So the proof of Theorem 1.1 is complete once we've shown the following:

Proposition 4.1. The sporadic simple groups and the Tits group admit an unmixed Beauville structure.

TABLE 4. Conjugacy classes for sporadic groups

\begin{tabular}{|l|rr||l|rr||l|rr|}
\hline$G$ & $C_{1}$ & $C_{2}$ & $G$ & $C_{1}$ & $C_{2}$ & $G$ & $C_{1}$ & $C_{2}$ \\
\hline \hline$M_{11}$ & $5 a$ & $8 a$ & $M_{24}$ & $11 a$ & $21 a$ & $H N$ & $22 a$ & $35 a$ \\
$M_{12}$ & $3 b$ & $10 a$ & $M c L$ & $9 a$ & $14 a$ & $L y$ & $31 a$ & $37 a$ \\
$J_{1}$ & $7 a$ & $11 a$ & $H e$ & $14 c$ & $15 a$ & $T h$ & $13 a$ & $31 a$ \\
$M_{22}$ & $7 a$ & $8 a$ & $R u$ & $16 a$ & $26 a$ & $F i_{23}$ & $13 a$ & $23 a$ \\
$J_{2}$ & $8 a$ & $15 a$ & $S u z$ & $11 a$ & $21 a$ & $C o_{1}$ & $23 a$ & $33 a$ \\
$M_{23}$ & $11 a$ & $14 a$ & $O N$ & $19 a$ & $16 a$ & $J_{4}$ & $31 a$ & $37 a$ \\
${ }^{2} F_{4}(2)^{\prime}$ & $10 a$ & $16 a$ & $C o_{3}$ & $21 a$ & $22 a$ & $F i_{24}^{\prime}$ & $23 a$ & $33 a$ \\
$H S$ & $7 a$ & $20 a$ & $C o_{2}$ & $11 a$ & $28 a$ & $B$ & $23 a$ & $31 a$ \\
$J_{3}$ & $12 a$ & $17 a$ & $F i_{22}$ & $21 a$ & $22 a$ & $M$ & $47 a$ & $59 a$ \\
\hline
\end{tabular}

Proof. In Table 4 we give for each sporadic group $G$ two conjugacy classes $C_{1}, C_{2}$ such that the structure constant $n\left(C_{1}, C_{1}, C_{2}\right)$ is non-zero and moreover no maximal subgroup of $G$ has non-trivial intersection with both classes. This is easily checked from the known lists of maximal subgroups, see [6] respectively the Atlas homepage. For the group $J_{2}$ we used explicit computation in the 6-dimensional representation over $\mathbb{F}_{4}$ and for ${ }^{2} F_{4}(2)^{\prime}$ in the permutation representation on 1600 points to check for generating triples. Since the element orders in the triples in Table 4 are prime to those in [22, Prop. 4.5], there do exist corresponding unmixed Beauville structures. 


\section{Bounds ON CHARACTER VALUES}

We will prove the following result which may be of independent interest.

Let $W$ be an irreducible Weyl group and $\rho$ a graph automorphism of its Dynkin diagram. Then there exists a constant $C=C(W, \rho)$ with the following property: whenever $\mathbf{G}$ is a connected reductive algebraic group with Weyl group $W=W(\mathbf{G})$ and $F: \mathbf{G} \rightarrow \mathbf{G}$ a Steinberg map inducing the graph automorphism $\rho$ on $W$, with group of fixed points $G:=\mathbf{G}^{F}$, then for every regular semisimple element $s \in G$ and any irreducible character $\chi \in \operatorname{Irr}(G)$ we have $|\chi(s)|<C$.

In fact we'll show a version which allows for $\mathbf{G}$ to be disconnected. Since we'll need some ingredients on characters of disconnected groups which are not yet available in the literature, we start by setting up some notation first.

Let $\mathbf{G}$ be an algebraic group with connected component of the identity $\mathbf{G}^{\circ}$. We assume throughout that $\mathbf{G} / \mathbf{G}^{\circ}$ is cyclic and that all elements of $\mathbf{G} / \mathbf{G}^{\circ}$ are semisimple. Let $F: \mathbf{G} \rightarrow \mathbf{G}$ be a Steinberg map on $\mathbf{G}$ with trivial action on $\mathbf{G} / \mathbf{G}^{\circ}$ and $G:=\mathbf{G}^{F}$, $G^{\circ}:=\left(\mathbf{G}^{\circ}\right)^{F}$. For $\mathbf{T}^{\circ}$ an $F$-stable maximal torus of $\mathbf{G}^{\circ}$ contained in a not necessarily $F$-stable Borel subgroup $\mathbf{B}^{\circ}$ of $\mathbf{G}^{\circ}$ we set $\mathbf{B}:=N_{\mathbf{G}}\left(\mathbf{B}^{\circ}\right)$ and $\mathbf{T}:=N_{\mathbf{B}}\left(\mathbf{T}^{\circ}\right)$. Following [9] we say that $\mathbf{T}$ is a maximal "torus" of $\mathbf{G}$ (note that this is not in general a torus!). Since all Borel subgroups of $\mathbf{G}^{\circ}$ and all maximal tori of $\mathbf{B}^{\circ}$ are conjugate in $\mathbf{G}^{\circ}$ respectively $\mathbf{B}^{\circ}$ we have $\mathbf{T} / \mathbf{T}^{\circ} \cong \mathbf{B} / \mathbf{B}^{\circ} \cong \mathbf{G} / \mathbf{G}^{\circ}$. Let $g \in \mathbf{T}$ generate $\mathbf{G} / \mathbf{G}^{\circ}$. Since by assumption $g^{-1} F(g) \in \mathbf{T}^{\circ}$ and $\mathbf{T}^{\circ}$ is connected, there exists by the theorem of Lang-Steinberg an element $h \in \mathbf{T}^{\circ}$ such that $g^{-1} F(g)=h^{-1} F(h)$, so that $\sigma:=g h^{-1} \in \mathbf{T}$ is $F$-stable and generates $\mathbf{G} / \mathbf{G}^{\circ}$, hence $G / G^{\circ}$.

For $\mathbf{U} \leq \mathbf{B}$ the unipotent radical of $\mathbf{B}$, define

$$
Y:=Y\left(\mathbf{U}^{F}\right):=\left\{x \in \mathbf{G} \mid x^{-1} F(x) \in \mathbf{U}\right\} .
$$

This variety has commuting actions of $G$ from the left and $T:=\mathbf{T}^{F}$ from the right by multiplication, so its $\ell$-adic cohomology groups with compact support $H_{c}^{i}(Y)$ are $G \times T$ bimodules. For $\theta \in \operatorname{Irr}(T)$ we let $H_{c}^{i}(Y)_{\theta}$ denote the $\theta$-isotypic component for the right $T$-action. Then the generalized character

$$
R_{T, \theta}(g):=\operatorname{tr}\left(g \mid H_{c}^{*}(Y)_{\theta}\right) \quad(g \in G)
$$

of $G$ constitutes an analogue of Deligne-Lusztig induction for the disconnected group $\mathbf{G}$ which has been studied by Digne-Michel [9]. We'll need the following property:

Proposition 5.1 (Disjointness). Let $\mathbf{T} \leq \mathbf{G}$ be a maximal "torus" of $\mathbf{G}, T:=\mathbf{T}^{F}$ and $\theta_{i} \in \operatorname{Irr}(T), i=1,2$. If the virtual $\mathbf{G}^{F}$-characters $R_{T, \theta_{1}}, R_{T, \theta_{2}}$ have an irreducible constituent in common, then there exists $g \in N_{\mathbf{G}}\left(\mathbf{T}^{\circ}\right)^{F}$ such that $\left.\theta_{1}^{g}\right|_{T^{\circ}}=\left.\theta_{2}\right|_{T^{\circ}}$.

Proof. When $\mathbf{G}$ is connected, this is well-known [8, Prop. 13.3]. We reduce to that case. By construction of $\sigma$, the set of powers $S:=\left\{\sigma^{j} \mid 0 \leq j \leq\left[\mathbf{G}: \mathbf{G}^{\circ}\right]-1\right\} \subset \mathbf{T}^{F}$ forms a system of coset representatives of $\mathbf{G} / \mathbf{G}^{\circ}$.

The Deligne-Lusztig variety $Y$ then decomposes into a disjoint union of open and closed subsets

$$
Y=\coprod_{g \in S} Y_{g}, \quad \text { where } Y_{g}:=\left\{g x \in g \mathbf{G}^{\circ} \mid x^{-1} F(x) \in \mathbf{U}\right\}
$$


SO

$$
H_{c}^{i}(Y)=\bigoplus_{g \in S} H_{c}^{i}\left(Y_{g}\right)
$$

by [8, Prop. 10.7(ii)]. Note that $Y_{g}$ is isomorphic to $Y_{1}$ via

$$
Y_{1} \longrightarrow Y_{g}, \quad x \mapsto g x
$$

Here, $Y_{1}$ is just the ordinary Deligne-Lusztig variety for the torus $T^{\circ}$ in the connected group $\mathbf{G}^{\circ}$. Moreover, $h \in G^{\circ}$ acts on $Y_{g}$ on the left as $h^{g}$ does on $Y_{1}$, and the right $T^{\circ}$-actions on $Y_{1}, Y_{g}$ commute with the above isomorphism.

If $H_{c}^{i}(Y)_{\theta_{1}}$ and $H_{c}^{j}(Y)_{\theta_{2}}$ have a common $\mathbf{G}^{F}$-constituent, then clearly there is also a common $G^{\circ}$-constituent of $H_{c}^{i}\left(Y_{g}\right)_{\theta_{1}}$ and $H_{c}^{j}\left(Y_{h}\right)_{\theta_{2}}$ for some $g, h \in S$. But then by [8, Prop. 13.3] the pairs $\left(\mathbf{T}^{\circ}, \theta_{1}^{g}\right),\left(\mathbf{T}^{\circ}, \theta_{2}^{h}\right)$ are geometrically $\mathbf{G}$-conjugate, where we identify $\theta_{i}$ with its restriction to $T^{\circ}$. This is the claim.

For $\mathbf{T} \leq \mathbf{G}$ a maximal torus, let us set $T_{0}^{\circ}:=C_{\mathbf{T}}^{\circ}(\sigma)^{F}$ and $G_{0}:=C_{\mathbf{G}}^{\circ}(\sigma)^{F}$. Note that when $\mathbf{G}=\mathbf{G}^{\circ}$ is connected then $T_{0}^{\circ}=T$ and $G_{0}=G$.

Definition 5.2. A semisimple element $s \in \mathbf{G}$ is called regular if it lies in a unique maximal "torus" of $\mathbf{G}$, which happens if and only if its connected centralizer is a (true) torus of $\mathbf{G}^{\circ}$ (which, in general, will not be a maximal torus of $\mathbf{G}^{\circ}$.)

Lemma 5.3. Let $s \in G^{\circ} \sigma$ be regular semisimple in the maximal "torus" $T$, so $C:=$ $C_{G}(s)=C_{T}(s)=C_{T}(\sigma)$. Then the characteristic function of the $G$-conjugacy class of $s$ is given by

$$
\psi=\frac{1}{|C|} \sum_{\theta \in \hat{T}} \theta\left(s^{-1}\right) R_{T, \theta}
$$

where $\hat{T}:=\operatorname{Irr}(T)$.

Proof. In the connected case, this is just [5, Prop. 7.5.5]. We mimic the proof given there. Let $\psi^{\prime}$ denote the characteristic function of $[s]$. The claim follows if we can show that $\left\langle\psi^{\prime}, \psi^{\prime}\right\rangle=\left\langle\psi, \psi^{\prime}\right\rangle=\langle\psi, \psi\rangle$. Clearly, $\left\langle\psi^{\prime}, \psi^{\prime}\right\rangle=1 /|C|$. Next.

$$
\left\langle\psi, \psi^{\prime}\right\rangle=\frac{1}{|G||C|} \sum_{\theta \in \hat{T}} \sum_{g \in[s]} \theta\left(s^{-1}\right) R_{T, \theta}(g)=\frac{1}{|C|^{2}} \sum_{\theta \in \hat{T}} \theta\left(s^{-1}\right) R_{T, \theta}(s) .
$$

In our situation where $s$ is semisimple regular, contained in the unique maximal "torus" $\mathbf{T}$, the character formula [9, Prop. 2.6] for $R_{T, \theta}$ takes the following form:

$$
R_{T, \theta}(s)=\frac{1}{|T|\left|C_{G}^{\circ}(s)\right|} \sum_{\left\{h \in G \mid s \in T^{h}\right\}}\left|C_{T}^{\circ}(s)\right| \theta\left(s^{h}\right)=\frac{1}{|T|} \sum_{h \in N_{G}(T)} \theta\left(s^{h}\right) .
$$

So

$$
\left\langle\psi, \psi^{\prime}\right\rangle=\frac{1}{|C|^{2}|T|} \sum_{h \in N_{G}(T)} \sum_{\theta \in \hat{T}} \theta\left(s^{-1}\right) \theta\left(s^{h}\right)=\frac{1}{|C|^{2}|T|} \sum_{\substack{h \in N_{G}(T) \\ s^{h} \sim s \text { in } T}}|\hat{T}|=\frac{1}{|C|}
$$

by the orthogonality relations for $\hat{T}$. Finally

$$
\langle\psi, \psi\rangle=\frac{1}{|C|^{2}} \sum_{\theta, \theta^{\prime} \in \hat{T}} \theta\left(s^{-1}\right) \theta^{\prime}(s)\left\langle R_{T, \theta}, R_{T, \theta^{\prime}}\right\rangle .
$$


But by [9, Prop. 4.8] we have

SO

$$
\left\langle R_{T, \theta}, R_{T, \theta^{\prime}}\right\rangle=\frac{1}{\left|T_{0}^{\circ}\right|}\left|\left\{g \in N_{G_{0}}(T) \mid{ }^{g} \theta=\theta^{\prime}\right\}\right|,
$$

$$
\langle\psi, \psi\rangle=\frac{1}{|C|^{2}\left|T_{0}\right|} \sum_{g \in N_{G_{0}}(T)} \sum_{\theta \in \hat{T}} \theta\left(s^{-1}\right)^{g} \theta(s)=\frac{1}{|C|^{2}\left|T_{0}\right|} \sum_{\substack{g \in N_{G_{0}}(T) \\ s \sim_{s}}}|C|=\frac{1}{|C|}
$$

Remember our standing assumptions that $\mathbf{G} / \mathbf{G}^{\circ}$ is cyclic and consists of semisimple elements and $F$ acts trivially on $\mathbf{G} / \mathbf{G}^{\circ}$. We now give a proof for an explicit bound on character values on regular semisimple elements (the key argument is taken from Malle $[38, \S 5]$ where the connected case is treated):

Theorem 5.4. Let $G=\mathbf{G}^{F}$ as above, $s \in G^{\circ} \sigma$ regular semisimple, lying in the (unique) maximal "torus" $\mathbf{T}$ of $\mathbf{G}$, and $\chi \in \operatorname{Irr}(G)$. Then

$$
|\chi(s)| \leq\left|W_{G}\left(\mathbf{T}^{\circ}\right)\right| \leq|W(\mathbf{G})|,
$$

where $W_{G}\left(\mathbf{T}^{\circ}\right):=N_{G}\left(\mathbf{T}^{\circ}\right) / T^{\circ}$ and $W(\mathbf{G}):=N_{\mathbf{G}}\left(\mathbf{T}^{\circ}\right) / \mathbf{T}^{\circ}$.

Proof. Let $C:=C_{G}(s)$. If $\psi$ denotes the characteristic function of the $G$-conjugacy class $[s]$ of $s$ then

$$
\langle\chi, \psi\rangle=\frac{1}{|G|} \sum_{g \in G} \chi(g) \psi\left(g^{-1}\right)=\chi(s)|[s]| /|G|=\chi(s) /|C|,
$$

so $\chi(s)=|C|\langle\chi, \psi\rangle$. With Lemma 5.3 this gives

$$
\chi(s)=|C|\langle\chi, \psi\rangle=\sum_{\theta \in \hat{T}} \theta\left(s^{-1}\right)\left\langle\chi, R_{T, \theta}\right\rangle .
$$

If $\left\langle\chi, R_{T, \theta}\right\rangle=0$ for all $\theta$ then $\chi(s)=0$ and the claim holds. So assume that there exists $\theta$ with $\left\langle\chi, R_{T, \theta}\right\rangle \neq 0$. Now, if $\theta^{\prime} \in \operatorname{Irr}(T)$ is such that $(\mathbf{T}, \theta)$ is not geometrically G-conjugate to $\left(\mathbf{T}, \theta^{\prime}\right)$, then $R_{T, \theta}, R_{T, \theta^{\prime}}$ do not have any constituent in common by Proposition 5.1. Thus there is at most one $N_{G}\left(\mathbf{T}^{\circ}\right)$-orbit $\Theta(\chi)$ on $\hat{T}$ such that $\chi$ occurs in $R_{T, \theta}$ for some $\theta \in \Theta(\chi)$. Also, as $\chi$ is irreducible,

$$
\left\langle\chi, R_{T, \theta}\right\rangle \leq\left\langle R_{T, \theta}, R_{T, \theta}\right\rangle^{\frac{1}{2}}=\left|W_{0}(\mathbf{T}, \theta)\right|^{\frac{1}{2}}
$$

(the last equality by [9, Prop. 4.8]), where

$$
W_{0}(\mathbf{T}, \theta):=\left\{w \in W_{0}(\mathbf{T}) \mid \theta^{w}=\theta\right\}, \quad W_{0}(\mathbf{T}):=N_{G_{0}}(\mathbf{T}) / T_{0}^{\circ} .
$$

Moreover, the orbit $\Theta(\chi)$ has length $\left[N_{G}\left(\mathbf{T}^{\circ}\right): N_{G}\left(\mathbf{T}^{\circ}, \theta\right)\right]=\left[W_{G}\left(\mathbf{T}^{\circ}\right): W_{G}\left(\mathbf{T}^{\circ}, \theta\right)\right]$, and $|\theta(s)| \leq \theta(1) \leq\left[T:\left.T^{\circ}\right|^{\frac{1}{2}}=\left[G: G^{\circ}\right]^{\frac{1}{2}}\right.$, so that finally

$$
\begin{aligned}
|\chi(s)| & =|C| \cdot|\langle\chi, \psi\rangle|=\left|\sum_{\theta \in \Theta(\chi)} \theta(s)^{-1}\left\langle\chi, R_{T, \theta}\right\rangle\right| \\
& \leq \sum_{\theta \in \Theta(\chi)}\left|\theta(s)^{-1}\right|\left|W_{0}(\mathbf{T}, \theta)\right|^{\frac{1}{2}} \leq|\Theta(\chi)| \cdot\left[G: G^{\circ}\right]^{\frac{1}{2}}\left|W_{0}(\mathbf{T}, \theta)\right|^{\frac{1}{2}} \\
& =\left|W_{G}\left(\mathbf{T}^{\circ}\right)\right| \cdot\left|W_{G}(\mathbf{T}, \theta)\right|^{\frac{1}{2}} / W_{G}\left(\mathbf{T}^{\circ}, \theta\right) \leq\left|W_{G}\left(\mathbf{T}^{\circ}\right)\right|
\end{aligned}
$$


for any $\theta \in \Theta(\chi)$.

The second to last term in the previous inequality is even slightly better than our claim whenever $\theta$ is not in general position (i.e., when $\chi$ is not an irreducible Deligne-Lusztig character $\left.\pm R_{T, \theta}\right)$.

Remark 5.5. Assume that $\mathbf{G}=\mathbf{G}^{\circ}$ is connected.

(a) If $\chi$ is unipotent, so contained in some $R_{T, 1}$, then $W(\mathbf{T}, \theta)=W_{G}(\mathbf{T})$ and we obtain the bound $|\chi(s)| \leq\left|W_{G}(\mathbf{T})\right|^{\frac{1}{2}}$.

(b) Since there are roughly $|T| /\left|W_{G}(\mathbf{T})\right|$ characters not vanishing on a regular element $t \in T$, and $\left|C_{G}(t)\right|=|T|$, one might expect an average character value of $\left|W_{G}(\mathbf{T})\right|^{\frac{1}{2}}$ on $t$. The example of $G=\mathrm{SL}_{2}(q)$ shows that character values will be larger than this: there exist irreducible characters which on regular semisimple elements of order $q+1$ take value $\zeta+\zeta^{-1}, \zeta$ a $q+1$ st root of unity. This has absolute value arbitrarily close to $2=\left|W_{G}(\mathbf{T})\right|$.

(c) See also [16, Thm. 3] for an elementary proof of a result that still gives an explicit (but worse) bound.

For truly disconnected groups we expect that the correct upper bound should be $\left|W_{0}(\mathbf{T})\right|$; this would follow from the above proof once a stronger disjointness statement than the one in Proposition 5.1 has been established, which compares characters of $T$ instead of their restrictions to $T^{\circ}$.

\section{Algebraic Groups}

We now consider triples in simple algebraic groups. For notational convenience, we now use standard font letters $G$ to denote algebraic groups, and $G(q)$ for the fixed groups under Frobenius endomorphisms with respect to an $\mathbb{F}_{q}$-rational structure.

Fix a prime $p$ and let $k$ be the algebraic closure of $\mathbb{F}_{p}$. Let $G$ be a simple algebraic group over $k$. Of course, since $G$ is locally finite, we cannot expect to generate $G$ with a finite number of elements. The replacement for generation is the property of generating the group $G(q)$ over a finite field $\mathbb{F}_{q}$ for arbitrarily large $q$.

We do have to exclude $G=\mathrm{SL}_{2}$. In this case, there is a strong rigidity result (see e.g. 37]) and the conclusion of Theorem 6.11 fails.

We first need a result about maximal subgroups of simple algebraic groups.

Lemma 6.1. Let $G$ be a simple simply connected algebraic group over $k$. There exist positive integers $m$ and $m^{\prime}$ (depending only upon the rank of the group) such that for any proper closed subgroup $H$ of $G$ one of the following holds:

(1) $H$ is contained in a (maximal) positive dimensional subgroup;

(2) $H$ is not contained in any proper positive dimensional closed subgroup and $|H| \leq$ $m$; or

(3) $H$ contains $G(q)$ for some prime power $q>m^{\prime}$ (including the possibility of a twisted form).

Moreover, there are only finitely many conjugacy classes of subgroups in (1) or (2).

Proof. If $G$ is classical, this follows from Aschbacher's theorem on maximal subgroups and representation theory. If $G$ is exceptional, then the result follows by the description 
of closed maximal subgroups (and maximal Lie primitive groups) in Liebeck-Seitz [32]. The finiteness of the number of conjugacy classes follows by [32] for (1) and by Martin [41, Prop. 1.4] for (2).

We next define some subvarieties of $G^{3}$. For conjugacy classes $C_{1}, C_{2}$ and $C_{3}$ in $G$ let

$$
V\left(C_{1}, C_{2}, C_{3}\right)=\left\{\left(x_{1}, x_{2}, x_{3}\right) \mid x_{i} \in C_{i}, x_{1} x_{2} x_{3}=1\right\} .
$$

For $M$ a subgroup of $G$ we set $V_{M}\left(C_{1}, C_{2}, C_{3}\right)=V\left(C_{1}, C_{2}, C_{3}\right) \cap M^{3}$ and

$$
V(M)=\bigcup_{g \in G}\left\{(x, y, z) \in G^{3} \mid x y z=1,\langle x, y\rangle \leq M^{g}\right\} .
$$

We need a result about dimensions of centralizers. Recall that an algebraic group is called reductive if it has no positive-dimensional closed connected unipotent normal subgroup.

Lemma 6.2. Let $G$ be a reductive algebraic group with $G^{\circ}$ non-abelian. If $g \in G$, then $\operatorname{dim} C_{G}(g)>0$.

Note that a reductive group with $G^{\circ}$ non-abelian is necessarily non-solvable, so the claim is just [44, Cor. 10.12] (as was kindly pointed out to us by T. Springer).

We also need the following fact (see [21, Th. 1.2] for a closely related result where $M$ is assumed to be reductive but $C$ is arbitrary):

Lemma 6.3. Let $G$ be an algebraic group, $M$ a closed subgroup, $C$ a semisimple conjugacy class of $G$. Then $C \cap M$ is a finite union of $M$-classes.

Proof. It suffices to assume that $M / M^{\circ}$ is cyclic of order $d$ prime to the characteristic (by considering the finitely many cosets $x M^{\circ}$ where $x$ is semisimple). By a result of Steinberg (see [9, Prop. 1.3]) every semisimple element of $M$ normalizes a maximal torus $T$ of $M^{\circ}$. Since all maximal tori of $M^{\circ}$ are conjugate, every element of $C \cap M$ is conjugate in $M$ to an element of $N_{M}(T)$. So if we can prove that $C \cap N_{M}(T)$ is a finite union of $N_{M}(T)$-classes, then clearly $C \cap M$ is a finite union of $M$-classes (indeed, at most the number of $C \cap N_{M}(T)$ classes). Thus it suffices to assume that $T$ is normal in $M^{\circ}$, whence $M^{\circ}=T \times U$ with $U$ unipotent.

Since $x$ is semisimple, $x^{d} \in T$. Thus, $D:=\langle T, x\rangle$ is a complement to $U$ in $M$. We claim that any two complements of $U$ in $M$ are conjugate in $M$. By induction on $\operatorname{dim} U$, it suffices to assume that $U$ is abelian (and of prime exponent if the characteristic is positive). Clearly, $T$ is contained in any complement of $U$. Thus, it suffices to observe that $H^{1}(D / T, U)=0$ (since $U$ is a projective $D / T$-module). As above, we may now assume that $M=D$ is reductive. Now we can apply [21, Th. 1.2] (or give a direct proof).

T. Springer has shown to us how this can also be obtained by a tangent space argument (similar to an argument of Richardson 42]).

We note a trivial bound:

Lemma 6.4. Let $G$ be a simple algebraic group, $M \leq G$ a closed proper subgroup. Let $C_{i}, 1 \leq i \leq 3$, be conjugacy classes of regular semisimple elements. 
(a) If $d$ is the minimal dimension of $C_{M}(x)$ for $x \in M$, then

$$
\operatorname{dim}\left(V\left(C_{1}, C_{2}, C_{3}\right) \cap V(M)\right) \leq \operatorname{dim} M+\operatorname{dim} G-2 d .
$$

(b) In particular, if $M^{\circ}$ is nonabelian and reductive, then

$$
\operatorname{dim}\left(V\left(C_{1}, C_{2}, C_{3}\right) \cap V(M)\right) \leq \operatorname{dim} M+\operatorname{dim} G-2 .
$$

Proof. Conjugation defines a surjective morphism $f: G \times W \rightarrow V\left(C_{1}, C_{2}, C_{3}\right) \cap V(M)$, where $W=V_{M}\left(C_{1}, C_{2}, C_{3}\right)$.

Let $x \in W$. Then $f\left(u^{-1}, x^{u}\right)=x$ for every $u \in M$. Thus $\operatorname{dim} f^{-1}(x) \geq \operatorname{dim} M$ for every $x \in W$. Since every element of the image of $f$ is in the $G$-orbit of some element of $W$, each fiber of $f$ has dimension at least $\operatorname{dim} M$.

By Lemma 6.3 the intersection $C_{i} \cap M$ is a finite union of $M$-classes. Thus, $\operatorname{dim} C_{i} \cap M \leq$ $\operatorname{dim} M-d$. It follows that $\operatorname{dim} W \leq 2(\operatorname{dim} M-d)$ and so

$$
\operatorname{dim} V\left(C_{1}, C_{2}, C_{3}\right) \cap V(M) \leq \operatorname{dim} G+\operatorname{dim} W-\operatorname{dim} M \leq \operatorname{dim} G+\operatorname{dim} M-2 d
$$

as claimed in (a).

If $M^{\circ}$ is nonabelian and reductive, then every centralizer has positive dimension by Lemma 6.2. Thus, $d \geq 1$ and (b) follows from (a).

We can now show that $V\left(C_{1}, C_{2}, C_{3}\right)$ is irreducible of dimension $2 \operatorname{dim} G-3 r$. We thank Tonny Springer for pointing out the fact that any irreducible component of this variety has dimension at least that.

Theorem 6.5. Let $G$ be a simple simply connected algebraic group of rank $r$. Let $C_{i}$, $1 \leq i \leq 3$, be classes of regular semisimple elements of $G$. Then $V\left(C_{1}, C_{2}, C_{3}\right)$ is an irreducible variety of dimension $2 \operatorname{dim} G-3 r$.

Proof. We first observe that every irreducible component of $V$ has dimension at least $2 \operatorname{dim} G-3 r$. Let $W=G \times G \times G, X=C_{1} \times C_{2} \times C_{3} \subset W$ and $Y=\{(x, y, z) \in$ $W \mid x y z=1\}$. Then $\operatorname{dim} X=3 \operatorname{dim} G-3 r$ and $\operatorname{dim} Y=2 \operatorname{dim} G$. Since $X$ and $W$ are irreducible and $W$ is smooth and irreducible, it follows by [46, p. 146] that indeed every component of $V=X \cap Y$ has dimension at least $\operatorname{dim} X+\operatorname{dim} Y-\operatorname{dim} W=2 \operatorname{dim} G-3 r$.

If $r=1$, it is straightforward to compute directly (cf. [37]). Now assume that $r>1$.

Choose a large power $q$ of $p$ so that the $C_{i}$ are defined over $G(q)$. Now we count the $\mathbb{F}_{q}$-points of $V\left(C_{1}, C_{2}, C_{3}\right)$ using the character formula given in the introduction. We just use the following facts:

(a) $|\chi(x)| \leq c$ for some constant $c$ depending only on the rank of the group for any irreducible character $\chi$ of $G(q)$ and any regular semisimple element $x \in G(q)$, by Theorem 5.4:

(b) $\sum_{\chi} \chi(1)^{-1} \leq 1+O\left(q^{-1 / 2}\right)$ where the sum is over all irreducible characters of $G(q)$ (this follows easily from Deligne-Lusztig theory as well, see the proof of [34, Thm. 1.1], using that the Coxeter number of a simple algebraic group not of type $A_{1}$ is larger than 2); and

(c) $C_{i}(q)$ is a single $G(q)$ conjugacy class (since centralizers of semisimple elements in groups of simply connected type are connected, see for example [40, Thm. 14.16]). 
It follows that

$$
\left|V\left(C_{1}, C_{2}, C_{3}\right)(q)\right|=\frac{|G(q)|^{2}}{c_{1} c_{2} c_{3}}\left(1+O\left(q^{-1 / 2}\right)\right)=q^{2 \operatorname{dim} G-3 r}\left(1+O\left(q^{-1 / 2}\right)\right),
$$

where $c_{i}$ are the orders of centralizers in $G(q)$ of elements from $C_{i}$.

The Lang-Weil theorem [31] on the number of points of an irreducible variety over a finite field now shows that $V\left(C_{1}, C_{2}, C_{3}\right)$ is irreducible and of dimension as claimed.

If $G$ is not simply connected, a variant of the previous result is still true.

Corollary 6.6. Let $G$ be a simple algebraic group of rank $r$ and $C_{i}, 1 \leq i \leq 3$, classes of regular semisimple elements of $G$. Then:

(a) Every irreducible component of $V\left(C_{1}, C_{2}, C_{3}\right)$ has dimension $2 \operatorname{dim} G-3 r$.

(b) For $\pi: \hat{G} \rightarrow G$ a simply connected covering of $G$, with (finite, central) kernel $Z$, choose conjugacy classes $D_{i}, 1 \leq i \leq 3$, of $\hat{G}$ that project onto $C_{i}$. Let $Z_{i}=$ $\left\{z \in Z \mid z D_{i}=D_{i}\right\}$ and set $Z_{0}=Z_{1} Z_{2} Z_{3}$. Then the number of components of $V\left(C_{1}, C_{2}, C_{3}\right)$ is $\left[Z: Z_{0}\right]$.

Proof. For $z \in Z$, let $V(z)$ be the variety of triples in $D_{1} \times D_{2} \times D_{3}$ with product $z$. By the previous result, this is an irreducible variety of dimension $2 \operatorname{dim} G-3 r$ (this variety is isomorphic to $\left.V\left(D_{1}, D_{2}, z^{-1} D_{3}\right)\right)$. Let $X:=X\left(D_{1}, D_{2}, D_{3}\right)=\cup_{z} V(z)$. Observe that $\pi(X)=V\left(C_{1}, C_{2}, C_{3}\right)$.

Suppose that $\left(x_{1}, x_{2}, x_{3}\right) \in V\left(C_{1}, C_{2}, C_{3}\right)$. Choose $y_{i} \in D_{i}$ with $\pi\left(y_{i}\right)=x_{i}$. Thus, $\left(y_{1}, y_{2}, y_{3}\right) \in V(z)$ for some $z \in Z$. Indeed, we see that $\pi\left(V\left(z_{1}\right)\right)=\pi\left(V\left(z_{2}\right)\right)$ if and only if $z_{1} Z_{0}=z_{2} Z_{0}$, and that the $\pi\left(V\left(z_{i}\right)\right)$ are disjoint if $z_{1} Z_{0} \neq z_{2} Z_{0}$. Let $R$ be a set of coset representative for $Z / Z_{0}$. Thus, $V\left(C_{1}, C_{2}, C_{3}\right)$ is a disjoint union of the $\pi(V(z)), z \in R$. Since $\pi$ is a finite map, this implies by the previous result that $\operatorname{dim} V\left(D_{1}, D_{2}, D_{3}\right)=$ $\operatorname{dim} V\left(C_{1}, C_{2}, C_{3}\right)$ and that there are $\left[Z: Z_{0}\right]$ different components.

We next consider connected but not necessarily reductive groups $H$. Recall the notion of regular semisimple elements from Definition 5.2 .

Corollary 6.7. Let $H$ be a connected algebraic group, with unipotent radical $U$ and set $L=H / U$, a reductive group. Let $C_{i}, 1 \leq i \leq 3$, be regular semisimple conjugacy classes of $H$. Let $V=V\left(C_{1}, C_{2}, C_{3}\right)$ be nonempty. Then every irreducible component of $V$ has dimension $2 \operatorname{dim}[H, H]-3 r$ where $r$ is the rank of $[H, H] / U \cong[L, L]$.

Proof. We first claim that $\operatorname{dim} C_{H}(x)=r$ for $x \in C_{i}$. Since $x$ is regular semisimple, we see that $C_{U}(x)=1$ and $C_{H}(x) \cong C_{H / U}(x)$. Since $H / U$ is reductive, the claim follows.

Now argue (using the bound for the dimension of a component of an intersection) as in the proof of Theorem 6.5 to deduce that every irreducible component of $V$ has dimension at most $2 \operatorname{dim}[H, H]-3 r$.

We now prove the reverse inequality. If $U=1$, then the result is clear by the result for simple groups. Suppose that $x_{i} \in C_{i}$ with $x_{1} x_{2} x_{3} \in U$. Then we can choose $u_{i} \in U$ with $u_{1}$ and $u_{2}$ arbitrary and $u_{3}$ uniquely determined by $u_{1}, u_{2}$ such that $\prod_{i}\left(x_{i} u_{i}\right)=1$. Since $x_{i}$ is semisimple regular, $x_{i} u_{i} \in C_{i}$. Thus, the result follows by the reductive case.

There is a version of this for some disconnected groups as well. We will say an algebraic group is almost simple if its connected component is simple and contains its centralizer. 
The proof of the next result is identical to that of the upper bound in Theorem 6.5 using Theorem 5.4 .

Corollary 6.8. Let $G$ be a not necessarily connected almost simple algebraic group. Let $C_{i}, 1 \leq i \leq 3$, be conjugacy classes of regular semisimple elements of $G$. Then $\operatorname{dim} V\left(C_{1}, C_{2}, C_{3}\right) \leq 2 \operatorname{dim} G-\sum_{i=1}^{3}\left(\operatorname{dim} G-\operatorname{dim} C_{i}\right)$.

Note that the result applies equally well to the case that $G$ is (essentially) a direct product of such groups.

We only need to apply the corollary in a special case which depends upon the following result.

Lemma 6.9. Let $G$ be a disconnected algebraic group such that $G^{\circ}$ is simple and $G / G^{\circ}$ is generated by a graph automorphism $\tau$ of order $d$. Let $X=\tau G^{\circ}$. Then the minimum dimension of $C_{G}(x), x \in X$, is the number of orbits of $\tau$ on the Dynkin diagram of $G$. Moreover, a generic element of $X$ is semisimple regular.

Proof. By inspection, we can choose $\tau$ such that $C_{G}(\tau)^{\circ}$ is a simple algebraic group of rank equal to the number of orbits of $\tau$ on the Dynkin diagram, and $\tau$ centralizes a regular semisimple element $g \in T$ of order prime to $d$ in some $\tau$-stable maximal torus $T \leq G^{\circ}$. Thus, $(\tau g)^{d}=g^{d}$ is regular semisimple in $G^{\circ}$. It follows that $\left(\tau g^{\prime}\right)^{d}$ is regular semisimple for an open subvariety of $g^{\prime} \in T$ (and so also for $g^{\prime} \in G^{\circ}$ ), whence the second statement follows.

Let $f: G^{\circ} \times \tau T \rightarrow X$ be the conjugation map. Let $t \in T_{0}$ with $(\tau t)^{d}$ semisimple regular. Now for $t^{\prime} \in T$, if $f\left(g, \tau t^{\prime}\right)=g\left(\tau t^{\prime}\right) g^{-1}=\tau t$, then $\tau t^{\prime}$ and $\tau t$ have the same centralizer in $G^{0}$ (namely $T_{0}$ ) and so $g$ normalizes $T_{0}$ and so $T$. Thus, the generic fiber of $f$ has dimension equal to $\operatorname{dim} T$ and $f$ is a dominant map. This shows that for elements in a nonempty subvariety of $X$, the dimension of the centralizer is equal to that of $T_{0}$, whence that is the minimum dimension. This completes the proof.

We will also need the following result which is a version of [20, Thm. 3.3].

Theorem 6.10. Let $G$ be a simple algebraic group over an algebraically closed field $k$ of characteristic $p \geq 0$. Let $N$ be a positive integer.

(a) If $p=0$, then $\{(x, y) \in G \times G \mid G=\overline{\langle x, y\rangle}\}$ is a nonempty open subvariety of $G \times G$.

(b) If $p>0$, then $\left\{(x, y) \in G \times G \mid G(q)^{g} \leq \overline{\langle x, y\rangle}\right.$ for some $\left.g \in G, q>N\right\}$ is a nonempty open subvariety of $G \times G$.

Proof. We first give the proof for $p>0$. It suffices to assume that $k=\overline{\mathbb{F}_{p}}$. By GuralnickTiep [24, Thm. 11.7] for the classical groups and using Liebeck-Seitz [33] for the exceptional groups, there is a finite collection of rational $k G$-modules such that the only proper closed subgroups of $G$ irreducible on all of them are conjugates of $G(q)$ for some sufficiently large $q$ (this includes twisted versions). The set of pairs which are reducible on any finite collection of modules is a closed condition. Since $G(q)$ is 2-generated, the set is nonempty (one only needs to know this for some sufficiently large $q$ ).

If $p=0$, then no proper closed subgroup is irreducible on the collection of submodules given. Since one can easily find two elements which generate a dense subgroup, the result follows. 
Theorem 6.11. Assume that $G$ is a simple simply connected algebraic group of rank $r>1$ over the algebraic closure $k$ of $\mathbb{F}_{p}$. Let $C_{i}, 1 \leq i \leq 3$, be regular semisimple classes and $V=V\left(C_{1}, C_{2}, C_{3}\right)$. Assume also that $G \neq \mathrm{SL}_{3}$ if each $C_{i}$ consists of elements of order 3 modulo $Z(G)$. Fix a positive integer $m$. Then for a generic $x:=\left(x_{1}, x_{2}, x_{3}\right) \in V$ we have that $\left\langle x_{1}, x_{2}\right\rangle \geq G(q)$ for some $q>m$.

Proof. As noted above, the set of pairs $(u, v) \in G \times G$ such that $\langle u, v\rangle$ contains $G(q)$ for some $q>m$ is open in $G \times G$. Thus, if the result fails, it follows by Lemma 6.1 that for every $x \in V,\left\langle x_{1}, x_{2}\right\rangle \leq M$ for some closed subgroup $M$ either with $M$ a positive dimensional maximal closed subgroup of $G$ or with $|M| \leq m$ and $M$ not contained in any proper positive dimensional subgroup. There are only finitely many conjugacy classes of such $M$ as we have already noted in Lemma 6.1. Thus, $V$ is contained in the finite union of these $V(M)$. Since $V$ is irreducible by Theorem [6.5, this implies that $V$ is contained in the closure of $V(M)$ for some fixed $M$. As we have seen above, this implies that $2 \operatorname{dim} G-3 r=\operatorname{dim} V \leq \operatorname{dim} M+\operatorname{dim} G$, whence $\operatorname{dim} M \geq \operatorname{dim} G-3 r$. In particular, since $r>1$ this implies that $\operatorname{dim} M>0$, and even that $\operatorname{dim} M>r$ unless possibly $G=\mathrm{SL}_{3}$ where $r=2$.

Suppose that $M$ is a maximal subgroup that is connected (or more generally $M^{\circ}$ contains all semisimple elements of $M)$. It follows by Corollary 6.7 that $\operatorname{dim} V_{M}\left(C_{1}, C_{2}, C_{3}\right) \leq$ $2 \operatorname{dim}[M, M]-3 r_{1}$, where $r_{1}=\operatorname{rk}([M, M])$. Arguing as in the proof of Lemma 6.4, this implies that

$$
\begin{aligned}
2 \operatorname{dim} G-3 r=\operatorname{dim} V\left(C_{1}, C_{2}, C_{3}\right) & \leq(\operatorname{dim} G-\operatorname{dim} M)+\operatorname{dim} V_{M}\left(C_{1}, C_{2}, C_{3}\right) \\
& \leq \operatorname{dim} G-\operatorname{dim} M+2 \operatorname{dim}[M, M]-3 r_{1} .
\end{aligned}
$$

Thus $\operatorname{dim} G \leq 2 \operatorname{dim}[M, M]-\operatorname{dim} M-3 r_{1}+3 r$. This clearly cannot be the case if $r_{1}=r$. In particular, $M$ cannot be of type $D_{n}$ in $C_{n}$ in characteristic 2 . If $r_{1} \leq r-1$, this implies that $\operatorname{dim} G \leq 2 \operatorname{dim}[M, M]-\operatorname{dim} M+3$. If $M$ is not semisimple and $r_{1}=r-1$, this yields $\operatorname{dim} G \leq \operatorname{dim} M+1$ which cannot occur since $r>1$. If $M$ is semisimple of rank $r-1$, the inequality above gives $\operatorname{dim} G \leq \operatorname{dim} M+3$. There are no proper semisimple subgroups of codimension at most 3. Thus, we see that either $r_{1}<r-1$ or at least one of the classes $C_{i} \cap M$ is not contained in $M^{\circ}$. In particular, $M$ is reductive.

Now $C_{i} \cap M$ is a finite union of conjugacy classes of $M$ by Lemma 6.3. Thus,

$$
\operatorname{dim} V\left(C_{1}, C_{2}, C_{3}\right) \cap M^{3}=\operatorname{dim} V\left(D_{1}, D_{2}, D_{3}\right)
$$

where $D_{i}$ is a conjugacy class of $M$ with $D_{i} \subseteq C_{i} \cap M$.

Suppose that $M^{\circ}$ is a torus. As we have noted above, this implies that $r=2$ and $G=$ $\mathrm{SL}_{3}$. Indeed, arguing as above, we see that $\operatorname{dim} V\left(D_{1}, D_{2}, D_{3}\right)+6 \geq \operatorname{dim} V\left(C_{1}, C_{2}, C_{3}\right)=$ 10 or $\operatorname{dim} V\left(D_{1}, D_{2}, D_{3}\right) \geq 4$. Clearly, $\operatorname{dim} V\left(D_{1}, D_{2}, D_{3}\right) \leq \operatorname{dim} D_{1}+\operatorname{dim} D_{2}$. It follows that the $D_{i}$ have finite centralizer in $M^{\circ}$. The only possibility is that the $D_{i}$ consist of elements of order 3 , but this is excluded by hypothesis.

Thus, every element of $M$ has a positive dimensional centralizer in $M$ by Lemma 6.2. Then with Lemma 6.4(b) the argument above gives a bit more:

$$
\operatorname{dim} M \geq \operatorname{dim} G-3 r+2 \text {. }
$$

Consider the case that $G$ is classical. First suppose that $M$ is reducible on the natural module. Then $M$ must be the stabilizer of a nondegenerate space of dimension less than 
$1 / 2$ the dimension of the space and $\operatorname{dim} M \geq \operatorname{dim} G-3 r+2$. The only possibility is that $G$ is an orthogonal group, $M$ is the stabilizer of a nondegenerate 1-space and $p>2$ (if $p=2$, every semisimple element in $M$ is contained in $M^{\circ}$ and so the better inequality applies).

If $G$ is of type $D_{n}, n \geq 4$, then $M=2 \times M^{\circ}$ and so we see that $\operatorname{dim}\left(V \cap M^{3}\right) \leq$ $2 \operatorname{dim} M^{\circ}-3(n-1)$. Thus, arguing as above, $\operatorname{dim} M \geq \operatorname{dim} G-2$, a contradiction.

Suppose that $G$ is of type $B_{n}, n \geq 3$ and $M^{\circ}=D_{n}$. Then apply Corollary 6.8 and Lemma 6.9 and argue as above. This gives the inequality

$$
2 \operatorname{dim} G-3 n \leq \operatorname{dim} G-\operatorname{dim} M+2 \operatorname{dim} M-3(n-1)
$$

or $\operatorname{dim} G \leq \operatorname{dim} M+3$, a contradiction.

If $M$ is irreducible but not almost simple, then either the natural module is imprimitive, tensor decomposable or tensor induced. By inspection, the only example with $\operatorname{dim} M \geq$ $\operatorname{dim} G-3 r+2$ is for $G=\mathrm{Sp}_{4}$ and $M=\mathrm{SL}_{2} \succ 2$. If $x y z=1$ with $x, y, z \in M$, then at most two of the elements can live outside $M^{\circ}$. It follows that at least one of the three elements has a 2-dimensional centralizer in $M^{\circ}$, whence the argument above gives $\operatorname{dim} V\left(C_{1}, C_{2}, C_{3}\right) \cap M^{3} \leq \operatorname{dim} M-3$ and we obtain a contradiction.

Suppose that $M$ is almost simple. Using the bound $\operatorname{dim} M \geq \operatorname{dim} G-3 r+2$ eliminates almost all possibilities (by the results of Lübeck [35] where he explicitly computes all irreducible modules of dimension less than $\left.r_{M}^{3} / 8\right)$. The exceptions are the cases where $(M, G)=\left(\mathrm{SL}_{2}=\mathrm{SO}_{3}, \mathrm{SL}_{3}\right),(M, G)=\left(G_{2}, B_{3}\right)$ or $(M, G)=\left(\mathrm{Sp}_{n}, \mathrm{SL}_{n}\right)$ with $n=4,6$. In all these cases, $M$ is connected, so it follows that $\operatorname{dim} V(M) \leq \operatorname{dim} G+\operatorname{dim} M-3 r_{1}$ and this is sufficient to show that $\operatorname{dim} V(M)<\operatorname{dim} V$.

Now let $G$ be an exceptional group. By [32], it follows that $\operatorname{dim} M<\operatorname{dim} G-3 r+2$ for any maximal reductive subgroup of $G$, whence the result follows.

Remark 6.12. In the excluded case $G=\mathrm{SL}_{3}$ and $C_{i}$ containing elements of order 3 , the proof does not exclude that we might generate a subgroup of the normalizer of the torus. Indeed that's what happens since it is well-known (and easy to see) that the triangle group generated by three elements of order 3 is solvable.

Another application of the Lang-Weil theorem [31] gives:

Corollary 6.13. Let $G$ and the $C_{i}$ be as in Theorem 6.11. If the $C_{i}$ are defined over $\mathbb{F}_{q}$ with $q$ sufficiently large, then there exist $x_{i} \in C_{i}$ with product 1 which generate $G(q)$

Proof. By the previous results, we just have to count the number of triples in $V$ which are conjugate to a triple in some subfield group $G\left(q_{0}\right)$. It is easy to see that these do not contribute enough to affect the result.

Remark 6.14. It follows by an easy argument that the results extend to arbitrary fields (and so we obtain Theorem 1.2). Here is the sketch (we thank Michael Larsen for pointing this out to us).

Let $G$ be a simple simply connected algebraic group over an algebraically closed field $k$. Let $C_{i}, 1 \leq i \leq 3$, be semisimple regular conjugacy classes of $G$. Let $V=V\left(C_{1}, C_{2}, C_{3}\right)$ be the variety of triples $\left(x_{1}, x_{2}, x_{3}\right)$ with $x_{i} \in C_{i}$ and product 1 . Since the $C_{i}$ are semisimple classes this is a closed subvariety of $G \times G \times G$. Note that the argument given in the 
proof of Theorem 6.5 shows that every irreducible component of $V$ has dimension at least $2 \operatorname{dim} G-3 r$.

Note that $V$ is defined over some finitely generated subring $R$ of $k$. If $M$ is a generic maximal ideal of $R$, then the reduction of $V(R)$ modulo $M$ will have the same dimension as $V$ and the same number of irreducible components over the algebraic closure of $R / M$. Since $R / M$ is a finite field, the result now follows from Theorem 6.5.

The proof of Theorem 6.11 now goes through verbatim and so holds as stated for $k$ of positive characteristic with $C_{1}, C_{2}, C_{3}$ torsion classes of regular semsimple elements.

If the characteristic is 0 or one of the $C_{i}$ consists of classes of infinite order, the proof shows:

Theorem 6.15. Assume that $G$ is a simple simply connected algebraic group of rank at least 2 over an algebraically closed field $k$ of characteristic $p \geq 0$. Let $C_{i}, 1 \leq i \leq 3$ be semisimple regular conjugacy classes of $G$. Assume either that $p=0$ or one of the classes $C_{i}$ consists of elements of infinite order. If $\left(x_{1}, x_{2}, x_{3}\right)$ is a generic triple in $V\left(C_{1}, C_{2}, C_{3}\right)$, then $\left\langle x_{1}, x_{2}\right\rangle$ is Zariski dense in $G$.

In characteristic 0 , it follows that the set of triples in this variety that generate a dense subgroup contains a nonempty open subvariety.

We close this section by demonstrating how the result of Theorem 1.2 can be extended by using asymptotic estimates on character values:

Theorem 6.16. Let $G$ be a simple, simply connected algebraic group of exceptional type. Let $C_{1}$ and $C_{2}$ be conjugacy classes of regular semisimple elements in $G$. Let $C_{3}$ be a conjugacy class of $G$ such that $x_{3} \in C_{3}$ has centralizer dimension $\operatorname{dim} C_{G}\left(x_{3}\right) \leq d_{G}$ with $d_{G}$ as in Table 5. Then the closure of $V\left(C_{1}, C_{2}, C_{3}\right)$ is irreducible of dimension $2(\operatorname{dim} G-\operatorname{rk}(G))-\operatorname{dim} C_{G}\left(x_{3}\right)$.

TABLE 5. Bounds for centralizer dimensions and character degree polynomials

\begin{tabular}{|l|ccccc|}
\hline$G$ & $G_{2}$ & $F_{4}$ & $E_{6}$ & $E_{7}$ & $E_{8}$ \\
\hline \hline$d_{G}$ & 5 & 21 & 19 & 39 & 97 \\
$e_{G}$ & 5 & 15 & 16 & 27 & 57 \\
\hline
\end{tabular}

Proof. Let $V$ denote the closure of $V\left(C_{1}, C_{2}, C_{3}\right)$. First assume that we are over the algebraic closure of a finite field. For $q$ a prime power, let $G(q)$ denote the group of fixed points of $G$ under a standard Frobenius endomorphism $F$ of $G$ corresponding to an $\mathbb{F}_{q}$-rational structure on $G$. Choose $q$ such that the $C_{i}$ are all defined over $\mathbb{F}_{q}, x_{3} \in G(q)$ and that each component of $C_{G}\left(x_{3}\right)$ is invariant under $F$. Let $x_{i} \in C_{i}(q)$ for $i=1,2$.

We claim that $\left|V\left(C_{1}, C_{2}, C_{3}\right)(q)\right|=q^{m}(1+o(1))$, where $m=2(\operatorname{dim} G-\operatorname{rk}(G))-$ $\operatorname{dim} C_{G}\left(x_{3}\right)$.

By Lang's Theorem $C_{3}$ splits into $e$ classes in $G(q)$ where $e$ is the number of conjugacy classes of $A\left(x_{3}\right):=C_{G}\left(x_{3}\right) / C_{G}\left(x_{3}\right)^{\circ}$ (since $F$ acts trivially on the set of components, see e.g. [40, Thm. 21.11]). Let $u_{1}, \ldots, u_{e}$ be representatives for the conjugacy classes of $A\left(x_{3}\right)$. 
Let $d=\operatorname{dim} C_{G}\left(x_{3}\right)$. Then the sizes of the $G(q)$-conjugacy classes in $x^{G} \cap G(q)$ will be $a_{i}^{-1} q^{\operatorname{dim} G-d}+O\left(q^{\operatorname{dim} G-d-1}\right)(1 \leq i \leq e)$, where $a_{i}$ is the order of the centralizer of $u_{i}$. Note that $\sum a_{i}^{-1}=1$ ( since $A\left(x_{3}\right)$ is the disjoint union of its conjugacy classes).

Thus,

$$
\left|V\left(C_{1}, C_{2}, C_{3}\right)(q)\right|=\frac{\left|C_{1}(q)\right|\left|C_{2}(q)\right|}{|G(q)|^{2}} \sum_{i=1}^{e}\left|u_{i}^{G(q)}\right| \sum_{\chi \in \operatorname{Irr}(G)} \frac{\chi\left(x_{1}\right) \chi\left(x_{2}\right) \chi\left(u_{i}\right)}{\chi(1)} .
$$

Since $\sum a_{i}^{-1}=1$, the contribution from the trivial character is $q^{m}+O\left(q^{m-1}\right)$. We argue as in the proof of Theorem 6.5 and show that $\sum_{\chi \neq 1}\left|\chi\left(u_{i}\right)\right| \chi(1)^{-1} \leq o(1)$. Note that by the result of Gluck [18] $\left|\chi\left(u_{i}\right)\right| / \chi(1)=o(1)$ for all $1 \neq \chi \in \operatorname{Irr}(G(q))$ and $1 \neq x \in G(q)$, so in the above sum we may ignore any bounded number of non-trivial characters.

By Lusztig's Jordan decomposition of characters, $\operatorname{Irr}(G)$ is the disjoint union of Lusztig series, indexed by semisimple classes in the dual group $G^{*}(q)$, each of size bounded only in terms of $r=\operatorname{rk}(G)$. Since $G^{*}(q)$ has at most $c_{1} q^{r}$ semisimple conjugacy classes (see [40, Thm. 26.10]), for some $c_{1}>0$, we conclude that $\mid \operatorname{Irr}\left(G(q) \mid \leq c_{2} q^{r}\right.$. By the orthogonality relations we have $\left|\chi\left(x_{3}\right)\right| \leq c_{3} q^{d_{G} / 2}$ for all $\chi \in \operatorname{Irr}(G(q))$. The smallest character degree of $G(q)$ not lying in the Lusztig series of an isolated element is of the form $c_{4} q^{e_{G}}$ with $e_{G}$ as given in Table 5. for example by [36]. The claim now follows by Theorem 5.4 since $d_{G}<2\left(e_{G}-r\right)$.

Since $V\left(C_{1}, C_{2}, C_{3}\right)$ is open in $V$ (as $C_{3}$ is open in its closure, see e.g. [40, Prop. 5.4]), it follows that $|V(q)|=\left|V\left(C_{1}, C_{2}, C_{3}\right)(q)\right|+O\left(q^{m-1}\right)=q^{m}(1+o(1))$. We complete the proof arguing as we did in Theorem 6.5. By Lang-Weil [31] $V$ has exactly one irreducible component with dimension $m$ (and none of dimension greater than $m$ ). By [46, p. 146], every irreducible component has dimension at least $m$ and so the variety is irreducible.

The result for arbitrary algebraically closed fields follows as in Remark 6.14.

If $C_{3}$ is a semisimple class, the proof is a bit easier as $V\left(C_{1}, C_{2}, C_{3}\right)$ is closed and $C_{G}\left(x_{3}\right)$ is connected. Note that the only non-trivial semisimple elements in $E_{8}$ with centralizer dimension larger than 97 are involutions with centralizer of type $D_{8}$ or $E_{7} \times A_{1}$, and elements in a 1-dimensional torus $T_{1}$ with centralizer $T_{1} E_{7}$.

\section{Generating Conjugacy Classes}

We now return to finite groups. In the proof of Theorem 1.1, we showed that in many finite simple groups there exist conjugacy classes $C$ and $D$ such that $G$ is generated by any pair of elements in $C \times D$. Moreover, in almost all the cases $C$ and $D$ were $\operatorname{Aut}(G)$ invariant. We investigate this further in this section. We will prove the following version of Theorem 1.3

Theorem 7.1. Let $G$ be a finite almost simple group with socle $S$. There exist conjugacy classes $C$ and $D$ of $G$ such that $S \leq\langle c, d\rangle$ for all $(c, d) \in C \times D$. Moreover aside, from the cases $S=\mathrm{O}_{8}^{+}(q), q \leq 3$, we may take $C, D \subset S$. In all cases, we may assume that $D$ is contained in $S$.

If $G$ contains a triality automorphism of $S=\mathrm{O}_{8}^{+}(2)$, the classes $C$ and $D$ cannot both be chosen to be contained in $S$. It seems likely this is also true for $q=3$.

In particular, a special case of the result is the following: 
Corollary 7.2. Let $G$ be a finite simple group other than $\mathrm{O}_{8}^{+}(q), q \leq 3$. There exist subsets $C, D$ of $G$ each invariant under $\operatorname{Aut}(G)$ such that $S=\langle c, d\rangle$ for each $(c, d) \in C \times D$.

One way of producing such classes is rather obvious:

Lemma 7.3. Let $G$ be a finite group with $g \in G$. Assume that $g$ is contained in a unique maximal subgroup $M$ of $G$. Let $C=g^{G}$. Let $D$ be the set of derangements of $G$ in the permutation action on $G / M$. Then $G=\langle g, h\rangle$ for any $h \in D$.

Of course, $D$ is always nonempty (by the well-known observation of Jordan). Moreover, if the $G$-class of $M$ is $\operatorname{Aut}(G)$-invariant, then we can take $C=g^{\operatorname{Aut}(G)}$ and $D$ will also be Aut $(G)$-invariant. Such elements exist in many (but not all) finite simple groups.

We start the proof of Theorem 7.1 by showing that alternating groups satisfy the result.

Proposition 7.4. Let $G=\mathfrak{A}_{n}, n \geq 5$. There exist conjugacy classes $C, D$ of $\mathfrak{S}_{n}$ contained in $\mathfrak{A}_{n}$ such that $G=\langle x, y\rangle$ for any $x \in C, y \in D$.

Proof. First suppose that $n=4 m \geq 8$ is divisible by 4 . Let $g$ be a product of a $2 m+1$ cycle and a $2 m-1$ cycle. Thus, some power of $g$ is a $2 m-1$ cycle. Let $M$ be a maximal subgroup containing $g$. We show that $M$ must be intransitive. Since there is a unique intransitive such maximal subgroup, the result will follow by Lemma 7.3 .

Since $\operatorname{gcd}(2 m+1,2 m-1)=1, M$ cannot be transitive and imprimitive. If $M$ is primitive, it follows by Williamson [47] that $M$ cannot exist.

Next suppose that $n=2 m \geq 10$ with $m$ odd. Let $g$ be a product of disjoint cycles of lengths $m-2$ and $m+2$. Argue precisely as above.

If $n=6$, let $C$ be the set of all 5 -cycles and let $D$ be the class of elements of order 4 in $G$. The only maximal subgroups containing an element $g$ of order 5 are $\mathfrak{A}_{5}$ (two classes), none of which contain an element of order 4 (thus, $C$ and $D$ are $\operatorname{Aut}(G)$-invariant).

Now suppose that $n \geq 5$ is odd. Let $g$ be an $n$-cycle. If $n$ is prime, let $h$ be a 3 -cycle. Then $G=\langle g, h\rangle$ (since any primitive group containing a 3-cycle contains $G$ ). If $n$ is not prime, let $q$ be a prime with $n / 2<q<n-3$, which exists by Bertrand's postulate. Let $h$ be the product of a 3 -cycle and a $q$-cycle. Then $\langle g, h\rangle$ is clearly primitive and contains a 3-cycle, whence the claim follows.

Proposition 7.5. Let $G$ be a sporadic simple group. Then there exist $\operatorname{Aut}(G)$-classes $C, D$ of $G$ such that $G=\langle x, y\rangle$ for any $(x, y) \in C \times D$.

Proof. This follows by the proof of Proposition 4.1, except for $J_{2}$ and the Tits group. For the first group, all pairs $(x, y) \in 5 c \times 7 a$ generate, for ${ }^{2} F_{4}(2)^{\prime}$ the same is true for all pairs of elements from $13 a \times 16 a$, by [6].

It remains to consider the finite simple groups of Lie type.

Proposition 7.6. Theorem 7.1 holds for the simple linear groups $\mathrm{L}_{n}(q)$.

Proof. First consider $\mathrm{L}_{2}(q)$. The alternating groups $\mathrm{L}_{2}(4) \cong \mathrm{L}_{2}(5) \cong \mathfrak{A}_{5}$ and $\mathrm{L}_{2}(9) \cong \mathfrak{A}_{6}$ were treated in Proposition [7.4. For $q \geq 11$ let $C_{1}$ contain elements of order $(q+1) / d$ and $C_{2}$ elements of order $(q-1) / d$, where $d=\operatorname{gcd}(2, q-1)$. Then by the well-known classification of subgroups of $\mathrm{L}_{2}(q)$, any pair $(x, y) \in C_{1} \times C_{2}$ will generate. For $\mathrm{L}_{2}(7)$ we let $C_{2}$ be a class of 7 -elements instead. 
For $n \geq 3$ let $C_{1}$ contain elements of order $\left(q^{n}-1\right) /(q-1) / d$, and $C_{2}$ elements of order $\left(q^{n-1}-1\right) / d$, where $d=\operatorname{gcd}(n, q-1)$. If $n \geq 5$ then any pair from $C_{1} \times C_{2}$ generates by our Corollary [3.4, unless $(n, q) \in\{(6,2),(7,2)\}$ when one of the two Zsigmondy primes does not exist. In the first of these cases, the only proper overgroup of elements from $C_{2}$ is an end-node parabolic, while in the second the only proper overgroup of elements from $C_{1}$ is the normalizer of a Singer cycle, by [22, Lemmas 2.3 and 2.4], but neither contains elements of order $2^{6}-1$.

Similarly, for $3 \leq n \leq 4$ it follows from [22, Lemma 2.3] that any pair generates unless possibly $(n, q) \in\{(3,2),(3,4),(4,2),(4,3)\}$. The groups $\mathrm{L}_{3}(2) \cong \mathrm{L}_{2}(7)$ and $\mathrm{L}_{4}(2) \cong \mathfrak{A}_{8}$ were already considered before. According to [6] the group $\mathrm{L}_{3}(4)$ is generated by any pair of elements of orders 5 and 7 ; the group $\mathrm{L}_{4}(3)$ is generated by any pair of elements of orders 5 and 13 .

Proposition 7.7. Theorem 7.1 holds for the simple unitary groups $\mathrm{U}_{n}(q), n \geq 3$.

Proof. For $n \geq 8$ this was already shown in Proposition [3.6, using the two classes in Table 3. For $n \leq 7$ odd let $C_{1}$ contain elements of order $\left(q^{n}+1\right) /(q+1) / d$, where $d=\operatorname{gcd}(n, q+1)$. By [22, Lemma 2.5] the only maximal overgroup of such an element is the normalizer of the maximal torus of that order, in which case we're done by Lemma 7.3 , or $(n, q) \in\{(5,2),(3,3),(3,5)\}$. In the latter three groups, no maximal subgroup contains elements of orders 11 and 9,7 and 12 , respectively 7 and 8 .

For $n \leq 6$ even let $C_{1}$ contain elements of order $\left(q^{n-1}+1\right) / d$. By [22, Lemma 2.6] the only maximal overgroup of such an element is the normalizer of $\operatorname{SU}_{n-1}(q)$, or $(n, q) \in\{(4,2),(6,2),(4,3),(4,5)\}$. In these last four groups, no maximal subgroup contains elements of orders 5 and 9, 11 and 30, 7 and 9, respectively 7 and 13 .

Proposition 7.8. Theorem 7.1 holds for the symplectic groups $\mathrm{S}_{2 n}(q), n \geq 2$, q odd for $n \geq 3,(n, q) \neq(2,2),(2,3)$.

Proof. For $n \geq 3$ this was already shown in Proposition 3.7, using the two classes in Table 3, unless $(n, q)=(4,3)$. For $\mathrm{S}_{8}(3)$ there is only one class of maximal subgroups containing elements of order $\left(q^{4}+1\right) / 2$, viz. the normalizer of an extension field subgroup $\mathrm{S}_{4}(9)$, by [22, Lemma 2.8].

For $n=2$ let $C_{1}$ consist of elements of order $\left(q^{2}+1\right) / d, d=\operatorname{gcd}(2, q-1)$, and $C_{2}$ of regular semisimple elements of order $q+1$ with centralizer of order $(q+1)^{2} / d$. By [22, Lemma 2.8] no maximal subgroup can contain elements from both classes.

Proposition 7.9. Theorem 7.1 holds for the orthogonal groups $\mathrm{O}_{2 n+1}(q), n \geq 3$.

Proof. For $n \geq 7$ this was already shown in Proposition 3.8, using the two classes in Table 3. Now assume that $4 \leq n \leq 6$. We take $C_{1}$ to consist of regular semisimple elements of order $\left(q^{n}+1\right) / d, d=\operatorname{gcd}(2, q-1)$, and $C_{2}$ containing elements of order $\left(q^{n-1}+1\right)(q+1) / d$. By [22, Lemma 2.7 and 2.8] no maximal subgroup contains both types of elements, unless possibly when $n=4, q=2$, in which case there is no Zsigmondy prime for $\left(q^{n-1}+1\right) / d$. The only maximal subgroups of $\mathrm{O}_{9}(2)=\mathrm{S}_{8}(2)$ of order divisible by 17 are $\mathrm{O}_{8}^{-}(2) .2, \mathrm{~S}_{4}(4) .2$ and $\mathrm{L}_{2}(17)$. The latter two do not contain elements of order 20 , and the first only contains one class, while $\mathrm{O}_{9}(2)$ contains two such classes.

For $n=3$ let $C_{1}$ be a class of elements of order $\left(q^{3}+1\right) / d, d=\operatorname{gcd}(2, q-1)$, and $C_{2}$ a class of elements of order $\left(q^{3}-1\right) / d$. According to [22, Lemma 2.7 and 2.8] no 
maximal subgroup contains both types of elements, unless possibly $q \in\{2,3,4,5\}$. For $\mathrm{S}_{6}(4)=\mathrm{O}_{7}(4)$ and $\mathrm{O}_{7}(5)$ none of the additional maximal subgroups has elements of order $\left(q^{3}+1\right) / d$. No maximal subgroup of $\mathrm{S}_{6}(2)=\mathrm{O}_{7}(2)$ contains elements of orders 9 and 15, and no maximal subgroup of $\mathrm{O}_{7}(3)$ contains elements of orders 13 and 14 .

Proposition 7.10. Theorem 7.1 holds for the orthogonal groups $\mathrm{O}_{2 n}^{-}(q), n \geq 4$.

Proof. This was already shown in Proposition [3.9, using the two classes in Table 3, except when $(n, q) \in\{(4,2),(5,2),(6,2),(4,4)\}$. No maximal subgroup of $\mathrm{O}_{8}^{-}(2)$ has order divisible by both 7 and 17; no maximal subgroup of $\mathrm{O}_{10}^{-}(2)$ has order divisible by both 11 and 17; no maximal subgroup of $\mathrm{O}_{12}^{-}(2)$ has elements of order both 11 and 65 by 22 , Lemma 2.10]; no maximal subgroup of $\mathrm{O}_{8}^{-}(4)$ has order divisible by both 257 and 13 .

Proposition 7.11. Theorem 1.3 holds for the orthogonal groups $\mathrm{O}_{2 n}^{+}(q), n \geq 4$.

Proof. Let $C_{1}, C_{2}$ denote the conjugacy classes of $\mathrm{O}_{2 n}^{+}(q)$ chosen in the proof of Proposition 3.10. Then the claim follows for $n \geq 7$ (as well as for $n=5$ provided that $q \geq 5$ ). For $n=6$ let $C_{1}, C_{2}$ consist of elements with invariant subspaces of types $5^{-} \oplus 1^{-}$respectively $4^{-} \oplus 2^{-}$. Then any pair of elements from $C_{1} \times C_{2}$ acts irreducibly, and then by Corollary 3.4 they generate $G$.

For $n=5$ let $C_{1}, C_{2}$ contain elements with invariant subspaces of types $4^{-} \oplus 1^{-}$respectively $3^{-} \oplus 2^{-}$. Then we conclude as before unless possibly when $q=2$. No maximal subgroup of $\mathrm{O}_{10}^{+}(2)$ has order divisible by 17 and 31 .

So now assume that $n=4$. Let $C_{1}, C_{2}$ contain regular semisimple elements with invariant subspaces of types $3^{-} \oplus 1^{-}$respectively $2^{-} \oplus 2^{-}$. Such classes exist whenever $q \geq 4$. Let $H \leq G$ contain elements from both classes. Then clearly $H$ is irreducible on the natural module. Thus, by [22, Lemma 2.9] either $H$ is contained in the normalizer of $\mathrm{SU}_{4}(q)$, of $\mathrm{U}_{3}(q)$ or of $\operatorname{Spin}_{7}(q)$, or $q \in\{2,3,5\}$. The order of $\mathrm{U}_{3}(q)$ is not divisible by a Zsigmondy prime divisor of $q^{2}+1$. Regular semisimple elements of order $q^{2}+1$ in $\mathrm{SU}_{4}(q)$ and in $\operatorname{Spin}_{7}(q)$ have centralizer order divisible by $q-1$, while elements in $C_{2}$ have centralizer order dividing $\left(q^{2}+1\right)^{2}$. Thus, $H=G$ for $q \notin\{2,3,5\}$. For $q=5$ the only additional subgroup of order divisible by $13=\left(q^{2}+1\right) / 2$ is $2 .{ }^{2} B_{2}(8)$, but its order is prime to 3 .

So now assume that $q=3$. Suppose that $G$ is almost simple with socle $S=\mathrm{O}_{8}^{+}(3)$. Note that $S$ contains 3 conjugacy classes of elements of order 20. The Sylow 5-subgroups of each of these subgroups of order 20 are not conjugate in $S$. Thus, any subgroup of $S$ containing elements of order 20 in more than one class must contain a Sylow 5-subgroup of $S$. Thus by [6], the only maximal subgroups containing such elements are isomorphic to $M:=\left(\mathfrak{A}_{6} \times \mathfrak{A}_{6}\right) \cdot 2^{2}$. We claim that $M$ has a unique conjugacy class of elements of order 20. Note that in $\mathfrak{A}_{6} \times \mathfrak{A}_{6}$, there are 4 conjugacy classes of elements of order 20 . The centralizer of any of them in $M$ is contained in $\mathfrak{A}_{6} \times \mathfrak{A}_{6}$, whence these classes are fused in $M$. Note that $M$ embeds in $\mathfrak{S}_{6} 22$, whence all elements of order 20 are contained in $\mathfrak{A}_{6} \times \mathfrak{A}_{6}$. Thus, there are no maximal subgroups of $S$ containing two different conjugacy classes of elements of order 20.

If $|G: S|$ has order prime to 3, then there are at least two distinct $G$-classes of elements of order $20 \mathrm{in} S$. The result follows in this case. If 3 does divide $|G: S|$, then all three classes of elements of order 20 in $S$ are fused in $G$. Let $C$ be the set of all elements of 
order 20 in $S$. Let $D$ be the $G$-conjugacy class of an outer automorphism of order a power of 3. If $(c, d) \in C \times D$, then by the discussion above, $S=\left\langle c, c^{d}\right\rangle$, whence the result.

Finally, let $G=\mathrm{O}_{8}^{+}(2)$. It can be checked by a random computer search that there are no $\operatorname{Aut}(G)$-invariant subsets $C, D \subset G$ such that any pair from $C \times D$ generates. Now let $C, D$ be two distinct classes of elements of order 15 in $G$. These are fused under the triality automorphism. Note that the third powers of elements from $C, D$ are not conjugate either, so that any subgroup containing elements $x \in C$ and $y \in D$ must have order divisible by 25. According to the Atlas the only maximal subgroups with this property are three classes of subgroups $\left(\mathfrak{A}_{5} \times \mathfrak{A}_{5}\right) \cdot 2^{2}$, (conjugate under triality) and each intersects a unique class of 15 -elements of $G$. Hence $\langle x, y\rangle$ does not lie in any proper subgroup. Argue as in the case of $q=3$ to complete the proof.

Theorem 7.1 now follows by the propositions in this section.

A straightforward reduction to the almost simple case now yields Corollary 1.4.

\section{REFERENCES}

[1] H. H. Andersen, J. Jørgensen, P. Landrock, The projective indecomposable modules of $\mathrm{SL}\left(2, p^{n}\right)$. Proc. London Math. Soc. (3) 46 (1983), 38-52.

[2] I. Bauer, F. Catanese, F. Grunewald, Beauville surfaces without real structures. Pp. 1-42 in: Geometric methods in algebra and number theory, Progr. Math., vol. 235, Birkhäuser Boston, 2005.

[3] I. Bauer, F. Catanese, F. Grunewald, Chebycheff and Belyi polynomials, dessins d'enfants, Beauville surfaces and group theory. Mediterr. J. Math. 3 (2006), 121-146.

[4] E. Bertram, Even permutations as a product of two conjugate cycles. J. Combinatorial Theory Ser. A 12 (1972), 368-380.

[5] R. W. CARTER, Finite groups of Lie type. Conjugacy classes and complex characters. Wiley Classics Library. John Wiley \& Sons, Chichester, 1993.

[6] J.H. Conway, R.T. Curtis, S.P. Norton, R.A. Parker, R.A. Wilson, Atlas of Finite Groups. Clarendon Press, Oxford, 1985.

[7] B. N. Cooperstein, Maximal subgroups of $G_{2}\left(2^{n}\right)$. J. Algebra 70 (1981), 23-36.

[8] F. Digne, J. Michel, Representations of finite groups of Lie type. LMS Student Texts, 21. Cambridge University Press, Cambridge, 1991.

[9] F. Digne, J. Michel, Groupes réductifs non connexes. Ann. Sci. École Norm. Sup. (4) 27 (1994), 345-406.

[10] S. Dolfi, M. Herzog, C. Praeger, A new solvability criterion for finite groups. Preprint 2010.

[11] S. Dolfi, R. Guralnick, M. Herzog, C. Praeger, A new criterion for certain families of finite groups. Preprint.

[12] B. Fairbairn, K. Magaard, C. Parker, Generation of finite simple groups with an application to groups acting on Beauville surfaces. Preprint.

[13] Y. Fuertes, G. GonzÁlez-Diez, On Beauville structures on the groups $S_{n}$ and $A_{n}$, Math. Z. 264 (2010), 959-968.

[14] Y. Fuertes, G. Jones, Beauville surfaces and finite groups. Preprint.

[15] S. Garion, M. Penegini, New Beauville surfaces, moduli spaces and finite groups. Preprint.

[16] S. Garion, M. Larsen, A. Lubotzky, Beauville surfaces and finite simple groups. Preprint 2010.

[17] M. Geck, G. Hiss, F. Lübeck, G. Malle, G. Pfeiffer, CHEVIE - A system for computing and processing generic character tables for finite groups of Lie type, Weyl groups and Hecke algebras. Appl. Algebra Engrg. Comm. Comput. 7 (1996), 175-210.

[18] D. GLuCK, Character value estimates for nonsemisimple elements. J. Algebra 155 (1993), 221-237.

[19] R. Gow, Commutators in finite simple groups of Lie type. Bull. London Math. Soc. 32 (2000), $311-315$. 
[20] R. GuRalnick, Some applications of subgroup structure to probabilistic generation and covers of curves. Pp. 301-320 in: Algebraic groups and their representations (Cambridge, 1997), NATO Adv. Sci. Inst. Ser. C Math. Phys. Sci., 517, Kluwer Acad. Publ., Dordrecht, 1998.

[21] R. Guralnick, Intersections of conjugacy classes and subgroups of algebraic groups. Proc. Amer. Math. Soc. 135 (2007), 689-693.

[22] R. Guralnick, G. Malle, Products of conjugacy classes and fixed point spaces. Submitted.

[23] R. Guralnick, C. Praeger, T. Penttila, J. Saxl, Linear groups with orders having certain large prime divisors. Proc. London Math. Soc. 78 (1999), 167-214.

[24] R. Guralnick, P. Tiep, Symmetric powers and a problem of Kollár and Larsen. Invent. Math. 174 (2008), 505-554.

[25] W. M. Kantor, Subgroups of classical groups generated by long root elements. Trans. Amer. Math. Soc. 248 (1979), 347-379.

[26] W. M. Kantor, R. A. Liebler, The rank 3 permutation representations of the finite classical groups. Trans. Amer. Math. Soc. 271 (1982), 1-71.

[27] W. M. Kantor, A. Lubotzky, A. Shalev, Invariable generation and the Chebotarev invariant of a finite group, preprint.

[28] P. Kleidman, The maximal subgroups of the finite 8-dimensional orthogonal groups $P \Omega_{8}^{+}(q)$ and of their automorphism groups. J. Algebra 110 (1987), 173-242.

[29] P. Kleidman, The maximal subgroups of the Steinberg triality groups ${ }^{3} D_{4}(q)$ and of their automorphism groups. J. Algebra 115 (1988), 182-199.

[30] P. Kleidman, The maximal subgroups of the Chevalley groups $G_{2}(q)$ with $q$ odd, the Ree groups ${ }^{2} G_{2}(q)$, and their automorphism groups. J. Algebra 117 (1988), 30-71.

[31] S. Lang, A. WeIL, Number of points of varieties in finite fields. Amer. J. Math. 76 (1954), 819-827.

[32] M. Liebeck, G. Seitz, A survey of maximal subgroups of exceptional groups of Lie type. Pp. 139146 in: Groups, Combinatorics and Geometry (Durham, 2001), World Scientific, 2003.

[33] M. Liebeck, G. Seitz, Subgroups of exceptional algebraic groups which are irreducible on an adjoint or minimal module. J. Group Theory 7 (2004), 347-372.

[34] M. Liebeck, A. Shalev, Character degrees and random walks in finite groups of Lie type. Proc. London Math. Soc. 90 (2005), 61-86.

[35] F. LÜBECK, Small degree representations of finite Chevalley groups in defining characteristic. LMS J. Comput. Math. 4 (2001), 135-169.

[36] F. LÜBECK, Smallest degrees of representations of exceptional groups of Lie type, Comm. Algebra 29 (2001), 2147-2169.

[37] A. M. Macbeath, Generators of the linear fractional groups. Pp. 14-32 in: Proc. Sympos. Pure Math., Vol. XII, Houston, Tex., 1967, Amer. Math. Soc., Providence, R.I.

[38] G. Malle, Exzeptionelle Gruppen vom Lie-Typ als Galoisgruppen. Dissertation, Math. Fak. Univ. Karlsruhe (TH) 1986.

[39] G. Malle, The maximal subgroups of ${ }^{2} F_{4}\left(q^{2}\right)$. J. Algebra 139 (1991), 52-69.

[40] G. Malle, D. Testerman, Linear algebraic groups and finite groups of Lie type. Cambridge University Press, to appear.

[41] B. Martin, Reductive subgroups of reductive groups in nonzero characteristic. J. Algebra 262 (2003), 265-286.

[42] R. W. Richardson, Conjugacy classes in Lie algebras and algebraic groups. Ann. of Math. (2) 86 (1967), 1-15.

[43] B. SRinivasan, The characters of the finite symplectic group $\operatorname{Sp}(4, q)$. Trans. Amer. Math. Soc. 131 1968, 488-525.

[44] R. Steinberg, Endomorphisms of Linear Algebraic Groups. Memoirs Amer. Math. Soc., 80 (1968).

[45] Th. Weigel, Generation of exceptional groups of Lie-type. Geom. Dedicata 41 (1992), 63-87.

[46] A. WeIL, Foundations of Algebraic Geometry. Amer. Math. Soc. (1946).

[47] A. Williamson, On primitive permutation groups containing a cycle. Math. Z. 130 (1973), 159162. 
3620 S. Vermont Ave, Department of Mathematics, University of Southern California, Los Angeles, CA 90089-2532, USA.

E-mail address: guralnic@usc.edu

FB Mathematik, TU Kaiserslautern, Postfach 3049, 67653 Kaiserslautern, Germany.

E-mail address: malle@mathematik.uni-kl.de 